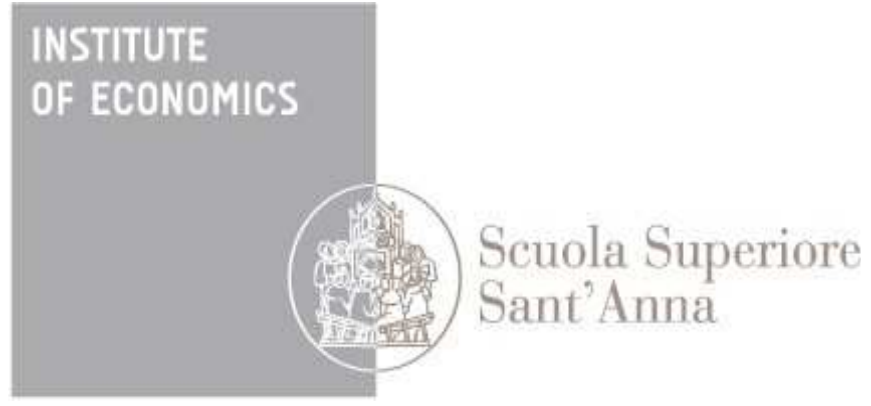

LEM | Laboratory of Economics and Management

Institute of Economics

Scuola Superiore Sant'Anna

Piazza Martiri della Libertà, 33 - 56127 Pisa, Italy ph. +3905088.33 .43$

institute.economics@sssup.it

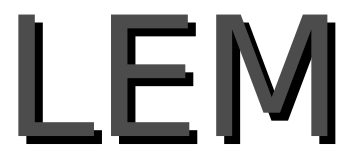

Working Paper Series

Boundedly Rational Opinion Dynamics in Social Networks: Does Indegree Matter?

Pietro Battiston ${ }^{\circ}$

Luca Stanca §

'Institute of Economics and LEM, Scuola Superiore Sant'Anna, Pisa, Italy

${ }^{\S}$ Department of Economics, University of Mialn Bicocca, Italy 


\title{
Boundedly Rational Opinion Dynamics in Social Networks: Does Indegree Matter?
}

\author{
Pietro Battiston* and Luca Stanca ${ }^{\dagger}$
}

January 22, 2015

\begin{abstract}
This paper investigates opinion dynamics and social influence in directed communication networks. We study the theoretical properties of a boundedly rational model of opinion formation in which individuals aggregate the information they receive from their neighbors by using weights that are a function of neighbors' indegree. We then present the results of a laboratory experiment explicitly designed to test the causal effect of indegree on social influence. We find that the social influence of an agent is positively affected by the number of individuals she listens to. When forming their opinions, agents take into account the structure of their communication network, although only to a limited extent.
\end{abstract}

Keywords: Social Networks, Learning, Social Influence, Bounded Rationality.

JEL Classification: D85, D83, A14, L14, Z13.

*Department of Economics, University of Milan Bicocca. Piazza dell'Ateneo Nuovo 1, 20126 Milan, Italy; Istituto di Economia, Scuola Superiore Sant'Anna, Piazza Martiri della Libertà 33, 56127 Pisa, Italy. E-mail: me@pietrobattiston.it

${ }^{\dagger}$ Corresponding author. Department of Economics, University of Milan Bicocca, Piazza dell'Ateneo Nuovo 1, 20126 Milan, Italy. E-mail: luca.stanca@unimib.it 


\section{Introduction}

Opinions are an important determinant of economic behavior, as they crucially affect the decisions of individual market participants. But how are opinions formed? Social learning plays a key role for opinion formation. Individuals update their opinions by observing the behavior of others and, above all, by communicating with the individuals they are connected to in their social network, such as family members, friends and colleagues. It is therefore important to understand how the structure of social relationships affects opinion dynamics. ${ }^{1}$ This paper provides a theoretical and empirical investigation of boundedly rational opinion formation in social networks.

The economic literature has recently paid increasing attention to opinion dynamics in social networks (see, e.g., Jackson and Yariv, 2010, Acemoglu and Ozdaglar, 2011, for comprehensive reviews). Two main approaches can be identified. A first group of studies focuses on Bayesian updating, under the assumption that agents optimally process the information about the network structure and the probability distribution of the signals observed by other agents (e.g., Gale and Kariv, 2003, Acemoglu et al., 2011, Acemoglu et al., 2014). Since Bayesian updating is generally characterized by high computational complexity, even under complete information, a second group of studies focuses on boundedly rational updating rules. These works generally consider simple and plausible protocols for belief updating, investigating the role played by the structure of the social network for the convergence of opinions, the efficient aggregation of information, and the social influence of individual agents.

The most common approach to boundedly rational opinion formation is based on the seminal model by DeGroot (1974), whereby agents update their opinion by taking an average of their neighbors' opinions. ${ }^{2}$ This basic framework has been widely adopted and extended, explicitly assuming a communication network and modelling opinions as point estimates rather than probability distributions (e.g., Bala and Goyal, 1998, Golub and Jackson, 2010, Möbius et al., 2010, Jadbabaie et al., 2012, Buechel et al., 2014). In a prominent paper, DeMarzo et al. (2003) start from a general model in which updating weights can differ among neighbors and change over time, to

\footnotetext{
${ }^{1}$ Understanding opinion dynamics in social networks has become even more relevant with the recent advent of communication technologies, such as Facebook and Twitter, that provide explicit representations of the previously implicit network of connections.

${ }^{2}$ DeGroot (1974) does not explicitly refer to a network environment (as French, 1956, and Harary, 1959, do in their studies on social power, later generalized by Friedkin and Johnsen, 1990), since all agents can communicate with all other agents. However, each individual places given weights on the opinions of others, so that the weights implicitly define a network. These weights, which are constant over time, determine the evolution and possible convergence of opinions.
} 
then focus on the case in which individuals place constant and equal weights on all neighbors. In this framework, failing to account for repetitions of information leads to persuasion bias: after repeated communication, opinions converge to a consensus that is biased towards the initial beliefs of the most influential (i.e., better connected) individuals. ${ }^{3}$ In this setting, agents' social influence depends on their position in the network and, more specifically, on being listened to, directly or indirectly, by many other agents.

In a recent paper, Corazzini et al. (2012) present an experimental investigation of persuasion bias in communication networks. Their results indicate that the structure of the network matters for social influence. More specifically, social influence is found to depend not only on being listened to by many others, consistent with persuasion bias, but also on listening to many others. In order to explain this finding, they propose a generalized boundedly rational updating rule according to which individuals place higher weight on the opinion of neighbors who have more sources of information. In this framework, the social influence of an agent can be positively affected by both her outdegree (the number of outgoing links) and her indegree (the number of incoming links), a feature referred to as "influential listeners".

It should be observed that Corazzini et al. (2012) proposed their generalized boundedly rational updating rule as an ex post interpretation of the results of an experiment aimed at testing persuasion bias, as predicted by DeMarzo et al. (2003). Since both outgoing and incoming links of the relevant nodes change at the same time, their experiment does not provide a test of a causal indegree effect, but only evidence consistent with it. In addition, despite providing a simple and plausible generalization, the influential listeners updating rule was proposed without an explicit characterization of its properties, such as convergence to a consensus (do individuals end up sharing the same opinion?) and efficiency (does such shared opinion optimally aggregate available information?). Therefore, building on Corazzini et al. (2012), the contribution of this paper is twofold.

First, we study theoretically the class of linear updating models and, in particular, the model proposed by Corazzini et al. (2012), as a simple generalization of the updating rule in DeMarzo et al. (2003). We obtain some positive and negative results on the feasibility of optimal rules of thumb, and characterize the way in which efficiency depends on the topology of the underlying network. We show that, in balanced networks (i.e., with indegree equal to outdegree for each node), placing higher weight on neighbors with higher indegree is less efficient than placing equal weights on all neighbors. On the other hand, in unbalanced networks it is generally more efficient to place higher weight on neighbors with higher indegree, and there exist

\footnotetext{
${ }^{3}$ See DellaVigna and Gentzkow (2010) for a recent survey of the evidence on persuasive communication.
} 
networks in which it is optimal to place arbitrarily high weight on agents with higher indegree.

Second, we present a laboratory experiment explicitly designed to test the causal effect of indegree on social influence. The structure of the directed network used in the experiment allows us to manipulate the indegree of the relevant nodes while keeping constant their outdegree, providing a clean test of the causal effect of indegree on opinion formation. Differently from previous experimental works, by comparing the social influence of different nodes we are able to test the null hypothesis that opinions are updated by averaging neighbors' opinions with equal weights, against the alternative that updating weights positively depend on neighbors' indegree. We find strong evidence of a causal indegree effect on opinion formation: the social influence of an agent is positively and significantly affected by the number of individuals she listens to. This is an important finding, as it indicates that, when forming their opinions, agents explicitly take into account the structure of their communication network, although only to a limited extent.

The remainder of the paper is structured as follows. Section 2 presents the theory (technical details are in Appendix A). Section 3 describes the experimental design (experimental instructions are in Appendix B). Section 4 provides the experimental results. Section 5 concludes with a discussion of the key findings.

\section{Theoretical Framework}

Following DeMarzo et al. (2003), consider a setting where a set $\mathcal{N}=\{1, \ldots, n\}$ of agents, communicating within a social network, want to estimate some unknown state of the world represented by the parameter $\theta \in \mathbb{R}$. Each agent starts with some initial information $x_{i}$ (henceforth referred to as a signal) about $\theta$. For simplicity, we assume that $x_{i}=\theta+\varepsilon_{i}$, with $\varepsilon_{i} \sim N\left(0, \sigma^{2}\right)$ independent across agents. The structure of the network is represented as a directed graph with adjacency matrix $q$, where $q_{i j}=1$ if agent $i$ listens to agent $j$, and 0 otherwise (we assume $q_{i i}=1$ for every $i$ ). ${ }^{4}$ We denote as $S(i) \subset \mathcal{N}$ the listening set of an individual $i$, that is, $j \in S(i) \Longleftrightarrow q_{i j}=1$. Communication takes place in discrete time: at each $t \geq 0$, agents report their current belief to their neighbors. Defining the vector of initial beliefs as $y^{0}=x$, we assume that, for each $t \geq 0$, agent $i$ updates her belief according to an updating rule

\footnotetext{
${ }^{4}$ Notice that in the graphical representations of networks presented below, an arrow from $i$ to $j$ means that agent $i$ talks to (rather than listens to) agent $j$, that is, $q_{j i}=1$. This is different from standard convention, but consistent with the direction of information flows and with the instructions of the experiment presented in Section 3.
} 


$$
y_{i}^{t+1}=f_{i}\left(y_{i_{1}}^{t}, \ldots, y_{i_{K}}^{t}\right)
$$

where $i_{1}, \ldots, i_{K}$ are the agents in $i$ 's listening set $S(i)$, including $i$. Once the network structure $q$, the updating rules $f_{1}, \ldots, f_{n}$, and the initial signals $x$ are determined, the evolution of opinions is obtained. Given an agent $i$, if $y_{i}^{\infty}=\lim _{t \rightarrow \infty} y_{i}^{t}$ exists, it will be referred to as her convergence or asymptotic belief. In what follows, we will be particularly interested in the case in which convergence beliefs exist and coincide for all $i$ : if this is the case, we will refer to such limit as the consensus belief, and denote it simply as $y^{\infty}$.

The foundations for this framework were laid by DeGroot (1974), who considers the case in which "opinions" are probability distributions rather than real numbers and $f$ is linear (in that context, $S(i)$ corresponds to $\{1, \ldots, n\})$ :

$$
y_{i}^{t+1}=\sum_{j \in S(i)} \pi_{i j} y_{j}^{t}
$$

where $\pi_{i j}$ is a constant representing the weight placed by agent $i$ on agent j. DeMarzo et al. (2003), following the studies on social power by French (1956) and Harary (1959), introduce an exogenously given network structure, and analyze in detail the specific updating rule

$$
y_{i}^{t+1}=\sum_{j \in S(i)} \frac{y_{j}^{t}}{|S(i)|} .
$$

One key feature of the updating rule in (2) is that agents do not take into account the entire structure of the network: they update their opinion by averaging all the opinions they get to know. While the rationale for this rule is its simplicity, the fact that individuals place the same weight on all neighbors rules out the possibility of placing higher weight on the opinions of some, possibly more informed, individuals. Consider instead a generalized boundedly rational (henceforth GBR) updating rule that is still linear, but with weights defined as follows:

$$
\pi_{i j}=\frac{q_{i j} d_{j}^{\rho}}{\sum_{h} q_{i h} d_{h}^{\rho}}
$$

where $d_{j}$ is agent $j$ 's indegree $\left(d_{j}=|S(j)|-1\right)$ and $\rho \in[0, \infty)$ is a fixed parameter. This rule, which was first introduced by Corazzini et al. (2012), provides a simple generalization of the updating rule in (2), while incorporating plausible and interesting features. Intuitively, when weighing the opinions of neighbors, agents attribute relatively more importance to 
those neighbors who have more direct sources of information, i.e., neighbors with higher indegree. ${ }^{5}$ To illustrate, let us consider some examples.

When $\rho=0$,

$$
\pi_{i j}=\frac{q_{i j}}{\sum_{h} q_{i h}}=\frac{q_{i j}}{|S(i)|},
$$

we obtain the updating rule in equation (2), as in DeMarzo et al. (2003): agents update their opinions placing equal weights on all neighbors.

When $\rho=1$,

$$
y_{i}^{t+1}=\frac{\sum_{j=1}^{n} q_{i j} d_{j} y_{j}^{t}}{\sum_{j=1}^{n} q_{i j} d_{j}},
$$

the opinion of each neighbor is weighted proportionally to her indegree.

In the limit case $\rho \rightarrow \infty$,

$$
y_{i}^{t+1}=\sum_{j \in \arg \max _{h} q_{i h} d_{h}} \frac{y_{j}^{t}}{\left|\arg \max _{h} q_{i h} d_{h}\right|},
$$

each agent only listens to the individual(s) with maximum indegree in her listening set.

DeMarzo et al. (2003) show that in a strongly connected network, i.e. in which every agent can communicate directly or indirectly with every other agent, ${ }^{6}$ any linear updating rule such that $\pi_{i j}>0$ whenever $q_{i j}>0$ guarantees convergence to a consensus belief. ${ }^{7}$ This implies that, in our setting, convergence is ensured for any $\rho \in[0, \infty) .{ }^{8}$ Moreover, if we rewrite equation (1) in matrix form as $y^{t+1}=\Pi y^{t}$, the vector of consensus beliefs $\bar{y}^{\infty}$ must satisfy the condition

$$
\bar{y}^{\infty}=\Pi \bar{y}^{\infty} .
$$

\footnotetext{
${ }^{5}$ It is also possible to consider a further generalization of the GBR rule, with updating weights that are both positively related to neighbors' indegree and negatively related to neighbors' outdegree (we thank an anonymous reviewer for this point). Although this negative outdegree effect can help to offset persuasion bias, it is harder to justify on behavioral grounds. While it is plausible that agents place higher weights on more informed neighbors, placing lower weights on neighbors who talk to many other agents is less plausible, as it requires a higher degree of sophistication.

${ }^{6}$ Formally, a network is strongly connected if for every pair of nodes $i, j$, it contains a path from $i$ to $j$.

${ }^{7}$ This is an application of their Theorem 1, in the special case where weights sum up to 1 and are constant over time.

${ }^{8}$ Theorem 1 by DeMarzo et al. (2003) does not apply to the limit case $\rho \rightarrow \infty$, since some neighbors can be attributed weights $\pi_{i j}=0$. Indeed, the assumption of positive weights cannot be dispensed with, as illustrated by the simple network with four nodes and $S(A)=\{B, D\}, S(B)=\{A\}, S(C)=\{B, D\}, S(D)=\{C\}$ : agents $A$ and $C$, who have maximum indegree and are not directly connected, will never change their own beliefs and therefore, assuming their initial signals differ, agreement will not be reached.
} 
i.e. $y^{\infty}$ is a right eigenvector of $\Pi$, with eigenvalue 1 . DeMarzo et al. (2003) also show that $y^{\infty}$ can be written as a weighted sum of the initial signals:

$$
y^{\infty}=\sum_{i=1}^{n} w_{i} x_{i},
$$

with $w$ being the unique (normalized) solution to

$$
w \Pi=w .
$$

If we consider $\Pi$ as the adjacency matrix of a weighted network, equation (8) can be interpreted as stating that the social influence $w_{i}$ of individual $i$ corresponds to her eigenvector centrality (Bonacich, 1972, Jackson, 2010).

Under the GBR updating rule, provided $\rho$ is not too large, social influence is positively related to outdegree (see Appendix A, Theorem A1): as in DeMarzo et al. (2003), an agent has higher influence if she is listened to by more agents. The relation between indegree and social influence results from the combination of two opposite effects, whose relative importance depends on the value of $\rho$. Intuitively, a higher indegree implies lower self-importance when updating opinions, thus decreasing social influence. On the other hand, for $\rho>0$, a higher indegree implies receiving a higher weight in others' (and own) opinions, thus increasing social influence. The larger $\rho$, the more likely it is that the latter effect prevails and the overall effect of indegree on social influence is positive (see Appendix A, Theorem A2). Therefore, whereas for $\rho=0$ social influence is related positively to outdegree and negatively to indegree, for sufficiently large values of $\rho$, social influence is positively related to both outdegree and indegree.

Let us now consider whether consensus beliefs are correct, in the sense of being optimal aggregates of agents' initial information. Given that all signals are equally informative, the consensus belief is correct if $w_{i}=\frac{1}{n} \forall i$, i.e.

$$
y^{\infty}=\frac{\sum_{i=1}^{n} x_{i}}{n}=\bar{x} .
$$

Different updating rules can therefore be compared by using the following measure of efficiency:

$$
E=-\sum_{i=1}^{n}\left(w_{i}-\frac{1}{n}\right)^{2}
$$

where $E=0$ if consensus beliefs are correct, while $E<0$ otherwise (notice $E \in(-1,0]){ }^{9}$

\footnotetext{
${ }^{9}$ For a given choice of $x_{1}, \ldots, x_{n}$, let $\delta=\mathbf{y}^{\infty}-\overline{\mathbf{x}}$. Maximizing efficiency corresponds to minimizing $|\delta|$. In order to define an absolute measure of efficiency, we observe that
} 
DeMarzo et al. (2003) analyzed the GBR rule under the restriction $\rho=0$, showing that it will not, in general, lead to a correct consensus in most network structures, as more connected agents have excessive social influence. When $\rho>0$, the GBR rule does not always neutralize such inefficiency: while it may lead to a consensus which is closer to the correct one than for $\rho=0$, this is not always the case. We thus start by asking, more generally, whether there exist rules of thumb that lead to correct beliefs for any given network structure. The answer is provided by the following two theorems.

Theorem 1 Given any strongly connected network $\overline{\mathcal{G}}$, there exists a linear updating rule $F_{\overline{\mathcal{G}}}$ which guarantees convergence to the correct consensus.

Proof. See Appendix A.

Definition 1 A linear updating rule is local if the weights applied by some agent $i$ are only determined depending on a subnetwork of limited diameter around $i$.

An implicit requirement for a "rule of thumb" is that it should not involve extended or complex computations: hence, subjects who are part of large networks will only be assumed to consider a limited subnetwork around them in order to decide what updating weights to adopt, rather than processing the entire network structure. This makes the following result particularly interesting.

Theorem 2 Given any local, linear updating rule $\bar{F}$, there exists a strongly connected network $\mathcal{G}_{\bar{F}}$ on which $\bar{F}$ does not guarantee convergence to the correct consensus.

Proof. See Appendix A.

While the result in Theorem 1 is positive, stating that for any given network it is possible to find an optimal linear rule, ${ }^{10}$ Theorem 2 ends the quest for the "perfect" rule of thumb: linear rules cannot at the same time be

$\delta$ is a linear combination of the initial deviations $x_{i}-\bar{x}$ with the elements of the vector $\Delta_{w}=\left(w_{1}-\frac{1}{n}, \ldots, w_{n}-\frac{1}{n}\right)$ as coefficients. $E$ is, up to a strictly monotonic transformation, the norm of $\Delta_{w}$. The specific transformation applied $\left(f(x)=-\left(x^{2}\right)\right)$ is irrelevant, since $E$ only has an ordinal interpretation.

${ }^{10}$ It is interesting to consider a constructive proof of Theorem 1: an algorithm which for any given network $\overline{\mathcal{G}}$ finds a linear rule which is efficient over it. Such an algorithm is described in Example 1 of Appendix A, and is based on the existence of a closed path $p_{n}$ passing through all nodes of the network (possibly more than once). Indeed, it clearly does not qualify as a "rule of thumb": in particular, the choice of $p_{n}$ requires significant ex-ante coordination among agents. 
simple and guarantee convergence to the correct consensus for any network structure, since finding optimal linear rules may require arbitrarily complex calculations.

Focusing on the GBR updating rule, for a given value of $\rho$ its efficiency will depend in a complex way on the topology of the network. In the following, we will study this relationship by focusing on some relevant classes of networks where the GBR rule displays particular features in terms of efficiency. We will denote as $E_{\rho}(\mathcal{G})$ the efficiency of the GBR rule when implemented over a network $\mathcal{G}$ with the given value of $\rho$, and as $\rho^{*}(\mathcal{G})$ the value of $\rho$ that maximizes $E_{\rho}(\mathcal{G})$ (when this value is unique). In all cases, it will be assumed that networks are strongly connected.

Let us define two nodes $(j, k)$ as equivalent if, after switching their labels, it is possible to arrange the other labels of the network in order to obtain an exact copy of the original network. In other words, two nodes are equivalent if the network structure around them is identical. Then, we can provide the following definitions:

Definition 2 A network structure is anonymous if all nodes are equivalent. $^{11}$

Definition 3 A belief updating rule $F$ is anonymous if all $f_{i}$ are symmetric in arguments $y_{j}^{t}$ and $y_{k}^{t}$, for any pair of equivalent nodes $j, k \neq i$.

In other words, a rule $F$ is anonymous if the labels of agents do not play any role. This is a basic condition for an analytical study of how the network topology affects opinion aggregation: it guarantees that simply relabeling the same network structure in a different way will not affect the dynamics of beliefs. The two definitions provided above allow us to state the following result, which generalizes Theorems 1 and 2 in French (1956), ${ }^{12}$ as well as Theorem 9 in Harary (1959).

Theorem 3 In any given anonymous, strongly connected network $\mathcal{G}$, if all agents play the same linear anonymous updating rule,

1. The beliefs in each period are uniquely determined by $\pi_{11}, \ldots, \pi_{n n}$, the self attributed weights,

\footnotetext{
${ }^{11}$ This is stronger than the concept of regular graph, which only considers the degree of vertices (rather than their position in the network), and corresponds instead to the concept of "automorphic group" employed by Harary (1959).

${ }^{12}$ While Theorem 2 by French (1956) is indeed a special case of our Theorem 3, his Theorem 1 additionally states that on complete networks, the consensus belief is reached in one step.
} 
2. The asymptotic consensus beliefs are correct: $w=\left(\frac{1}{n}, \ldots, \frac{1}{n}\right){ }^{13}$

Proof. See Appendix A.

Since the GBR updating rule is both linear and anonymous, a direct consequence of Theorem 3 is that, in an anonymous strongly connected network, opinions will converge to the true average of signals for any choice of $\rho$. For an example, see Figure 1, displaying a complete network with four nodes: since the value of $\rho$ is irrelevant, the efficiency of the GBR rule is constant. More specifically, since the consensus belief is correct, the efficiency is always equal to the maximum (i.e. 0 ).

Figure 1: An anonymous complete network
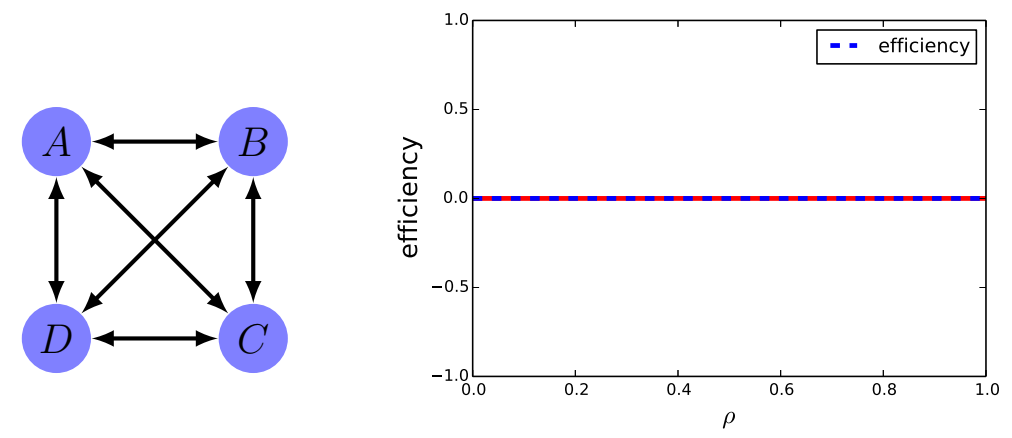

The requirement of anonymity of a network is a very strong one. In fact, the class of networks on which the GBR rule will perform identically for any given $\rho>0$ is significantly larger than that of anonymous ones. To see this, notice that on any regular network, ${ }^{14}$ independently of $\rho$, each agent will attribute the same importance to the opinion of each neighbor, since all of them will have the same indegree. Interestingly, the regularity of the network is also a necessary condition for $\rho$ to be irrelevant, as stated in the following theorem.

Theorem 4 On a strongly connected network $\mathcal{G}$, the convergence belief and social weights obtained under a GBR rule with given $\rho>0$ coincide with the ones obtained under $\rho=0$ if and only if $\mathcal{G}$ is regular.

\footnotetext{
${ }^{13}$ One may wonder if anonymity or linearity alone is a sufficient requirement for this theorem to hold. The answer is negative: two counter-examples are given, respectively, by the rules "weigh the opinion coming from the highest labeled neighbor as much as the average of all others" (which is linear but not anonymous) and "weigh the highest opinion coming from the neighbors as much as the average of all others" (which is anonymous but not linear).

${ }^{14} \mathrm{~A}$ directed network is regular if all nodes have the same indegree and outdegree (the two must necessarily coincide).
} 
Proof. See Appendix A.

Theorems 3 and 4 suggest that, for specific classes of networks, the GBR rule may be redundant: in the first case, because in anonymous networks any anonymous rule is equivalent, and in the second case because in regular networks the value of $\rho$ is irrelevant. The results that follow characterize wider classes of networks in which the GBR rule has instead non-trivial implications. We first focus on balanced networks.

Definition 4 A network is balanced if each node has indegree equal to outdegree. $^{15}$

Notice the inclusion hierarchy of the classes defined so far: all anonymous networks are regular, and all regular networks are balanced. In addition to that, the class of balanced networks also includes all undirected networks, that is, such that $q_{i j}=q_{j i}$ for all $i, j$.

In balanced networks, as shown below, the value of $\rho$ begins to matter.

Theorem 5 On any given balanced, strongly connected network $\mathcal{G}$, there is no $\rho>0$ which makes the GBR rule more efficient than $\rho=0$.

The proof is in Appendix A, but it can be interesting to consider an intuitive explanation. In this class of networks, the problem of persuasion bias is attenuated. Although it is present in the first period, in the long run agents with higher outdegree exploit their influence to convey richer information, since they also have higher indegree. Intuitively, their belief has higher weight, but their own initial opinion gets diluted in their belief. This does not occur when $\rho>0$, which causes agents with higher outdegree to place even higher weight on their own signal.

Together with Theorem 4, Theorem 5 establishes that on all networks which are balanced, strongly connected but not regular, any $\rho>0$ makes the GBR rule strictly less efficient than with $\rho=0$. As an example, consider the network structure in Figure 2. While it is not anonymous (nodes $B$ and $D$ are identical, but they differ from nodes $A$ and $C$ ), it shares with the complete network the feature of being undirected, and hence balanced. Figure 2 also shows that the optimal value of $\rho$ is 0 . Notice that Theorem 5 only holds asymptotically: it is easy to provide counter-examples in finite time, by replacing in the definition of efficiency (equation 10) the weights $w_{i}$ with those calculated after a finite number of periods.

\footnotetext{
${ }^{15}$ This definition is adopted from Olfati-Saber and Murray (2004). It is more general than the ones used by Corazzini et al. (2012) and DeMarzo et al. (2003), and corresponds to the concept of isograph used in graph theory. In particular, all weakly connected isographs are strongly connected and are also referred to as Eulerian digraphs (see Sridharan and Parthasarathy, 1972).
} 
Figure 2: An undirected, and hence balanced, network
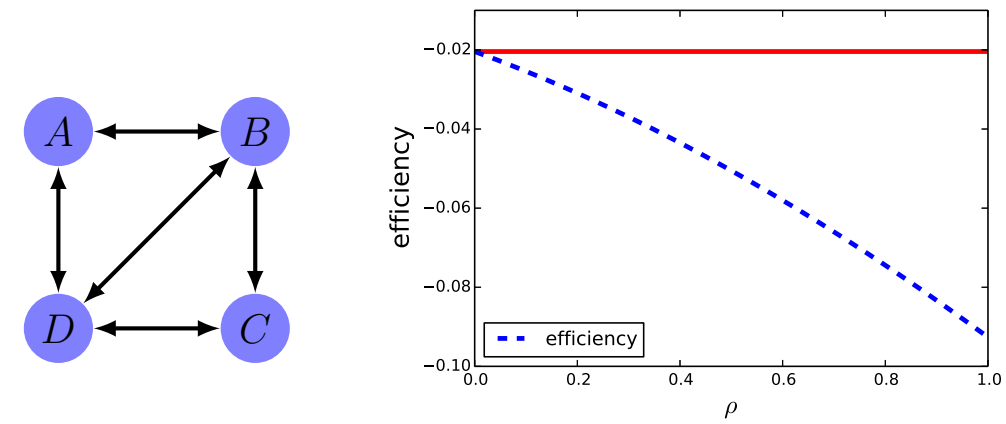

More importantly for our purposes, outside this specific class of networks things can change radically. The network presented in Figure 3, which is the one used in the experiment presented in Section 3, is unbalanced and characterized by an optimal value of $\rho$ that is positive (0.04). For higher values of $\rho$, the social influence of $B$, who has a higher indegree than $D$, increases, while the one of $D$ decreases. The following result shows that, by introducing even a small level of "unbalancedness" in an anonymous network, the optimal level of $\rho$ becomes positive.

Theorem 6 Given any regular network $\mathcal{G}$ and $i, j, h$ such that $q_{i j}=1$ and $q_{h j}=0$, let $\mathcal{G}^{\prime}$ be the result of setting $q_{i j}=0$ and $q_{h j}=1$. If $\mathcal{G}^{\prime}$ is strongly connected, $\rho^{*}\left(\mathcal{G}^{\prime}\right)>0$.

Proof. See Appendix A.

Figure 3: The network used in the experiment
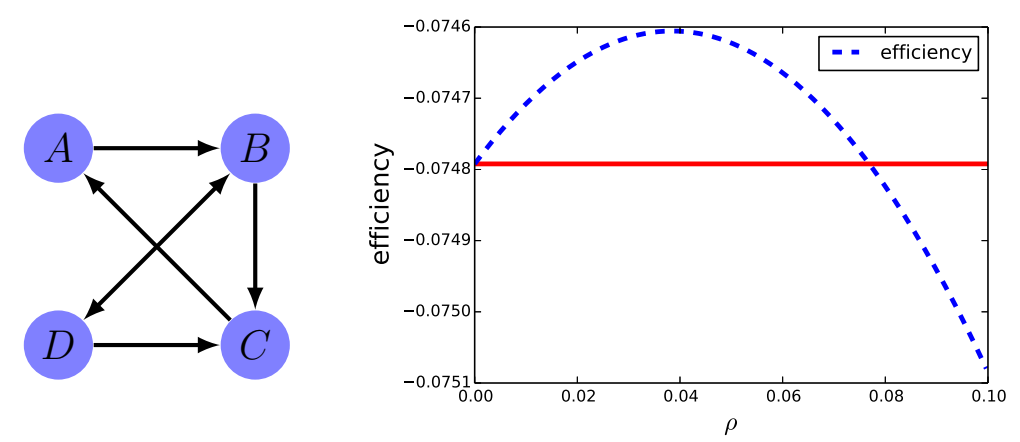

We already know from Theorem 3 that in a regular network, the counterbalancing action of the GBR rule is null. This result states that if instead we introduce even a minimal element of unbalancedness (in $\mathcal{G}^{\prime}$, the indegree 
of node $i$ is decreased by 1 , in favor of $h$ ), a strictly positive $\rho$ will allow to recover at least some of the lost efficiency. The following theorem states that no value of $\rho$ is in principle "too high".

Theorem 7 Given any $\bar{\rho} \in[0, \infty)$, there exists a strongly connected network $\overline{\mathcal{G}}$ such that $\rho^{*}(\overline{\mathcal{G}})>\bar{\rho}$.

Proof. See Appendix A.

In short, our results for the generalized boundedly rational updating rule can be summarized as follows. In regular networks, the value of $\rho$ is irrelevant, and in balanced, non-regular networks, a positive value of $\rho$ decreases efficiency. If a network is unbalanced, however, placing a higher weight on neighbors with higher indegree can lead to recover some of the efficiency loss, and there exist networks in which the optimal value of $\rho$ is arbitrarily high. Hence, the key feature of the GBR updating rule, that individuals may place higher weight on the opinions of more informed neighbors, is not only behaviorally plausible, but also generally efficient in unbalanced networks. In order to assess the empirical validity of the GBR rule, in the next Section we present an experimental test of the causal effect of indegree on opinion dynamics and social influence.

\section{Experimental Design}

The experiment is designed to test the effects of agents' position in a communication network on their social influence. More specifically, our experimental design allows us to manipulate agents' indegree without affecting their outdegree and the corresponding eigenvector centrality. Therefore, it enables us to provide a clean test of the effect of indegree on social influence, as predicted by the generalized boundedly rational updating rule studied in Section 2, that would be absent under either Bayesian updating or boundedly rational updating à la DeMarzo et al. (2003).

\subsection{Task}

At the beginning of the experimental task, individuals are anonymously matched in groups of four. In each group, subjects are connected through a communication network, and each subject is assigned a label $(A, B, C, D)$ that defines her position in the network. Each subject is assigned an integer number randomly drawn from a normal distribution, henceforth referred to 
as a signal, denoted with $x_{A}, x_{B}, x_{C}, x_{D}$, respectively. ${ }^{16}$ The task is based on a discrete time setting over 8 rounds. In each round the subjects are asked to guess the average $\bar{x}=\frac{x_{A}+x_{B}+x_{C}+x_{D}}{4}$ of the four signals in their group. The choice of replacing the population mean $(\theta)$ with the sample mean $(\bar{x})$ in the experimental setting is made to simplify the task, as it allows subjects to concentrate on aggregation of information rather than statistical inference.

In order to be able to update their beliefs, at the beginning of each round subjects receive information from the group members they are connected to. More specifically, at time $t$ each individual is informed about the guesses at time $t-1$ of the other group members connected to her (the network structure, that defines who receives information from whom, is described in the next subsection). Therefore, while in the first round subjects only directly know their own signal, over successive rounds they directly or indirectly receive information about the signals received by the other group members. If all four group members optimally process the information they receive, over successive rounds each of them can correctly guess $\bar{x}$.

The mechanism for eliciting beliefs is as follows: each individual is informed that at the end of the session, one round will be randomly extracted to determine earnings. Given the guess $y^{*}$ of the individual in that round, and the average of the signals in the group $(\bar{x})$, the individual's payoff is 15 euro minus the absolute difference between $y^{*}$ and $\bar{x}$, in addition to a show-up fee of 5 euros. This implies that individuals have an incentive to report in each round their best guess for the group average. We adopt an absolute deviation scoring rule for three reasons. First, a quadratic scoring rule, commonly used for belief elicitation, would substantially complicate the calculation of payoffs, hence increasing the likelihood of mistakes due to miscomprehensions. Second, for a given average gain, a quadratic scoring rule would increase the likelihood of earning very small payoffs, thus adversely affecting the incentive to exert effort in the task. Third, given normality of signals, our setting is perfectly symmetric, so that the median of the posterior's distribution coincides with the mean, and although we cannot directly control subjective probability distributions, we can test ex post for systematic biases in expressed beliefs. In addition, experimental subjects were explicitly instructed to update their beliefs by averaging all known and inferred signals, and control questions indicated that this instruction was clearly understood.

\footnotetext{
${ }^{16}$ The four signals for each group/phase were extracted as follows. An integer number $\theta$ was extracted from a uniform distribution in a range between 200 and 800 . Four positive integers were then randomly drawn from a normal distribution with mean $\theta$ and variance 100. The signals ranged between 219 and 790 .
} 


\subsection{Treatments}

Figure 4 describes the strongly connected directed network structure we use in the experiment. The number of nodes is small in order to provide a simple setting for the experimental subjects, but at the same time sufficiently large to imply interesting opinion dynamics. The network structure implies that $A$ is informed about past beliefs of $C, B$ is informed about past beliefs of $A$ and $D, C$ is informed about past beliefs of $B$ and $D$, while $D$ is informed about past beliefs of $B$. The indegree and outdegree of the four nodes are $A=(1,1), B=(2,2), C=(2,1), D=(1,2)$, respectively. The reason for choosing this specific network structure is that, as explained below, it allows us to cleanly test the key hypotheses of the experiment.

Figure 4: Structure of the communication network

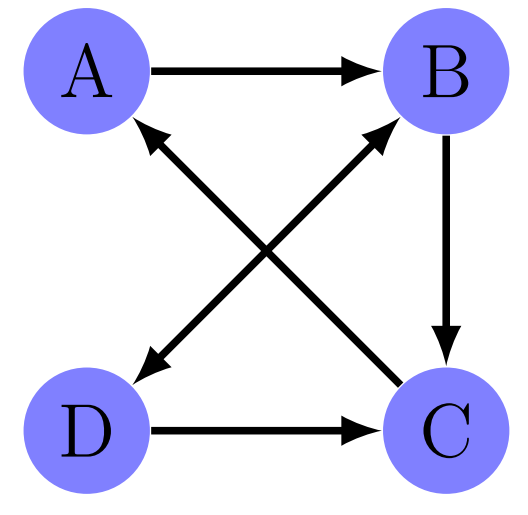

The treatment variable is the node that the subject is assigned to within the network. The four treatments (nodes $A, B, C$ and $D$ ) are implemented in a within-subjects design. This means that, in an experimental session, each subject performs the task four times, thus taking part in four subsequent phases of 8 rounds (32 rounds overall). In each of the four phases, each subject is randomly assigned to a different node (position) in the network. Therefore, within a session, each individual is assigned to each node in exactly one of the four phases. ${ }^{17}$ Subjects receive a different set of signals at the beginning of each phase, while the composition of the groups is unchanged throughout the four phases.

Since we aim at assessing if and how agents' social influence is affected by their position in the network, it is important to control for the possible confounding effects of the labels attached to each node $(A, B, C, D)$ and of subjects' visual location in the network (upper left, upper right, bottom

\footnotetext{
${ }^{17}$ It should be noted that our within-subjects design, where the treatment variable is the node that the subject is assigned to within the social network, allows us to compare the social influence of agents at different nodes while eliminating the effects of individual-level heterogeneity in updating.
} 
left, bottom right). It is possible, for instance, that subjects tend to give more importance (higher weight) to nodes denoted by letters that come first in the alphabet (e.g. $A$ vs $D$ ). Similarly, subjects might tend to give more importance to nodes located in the top-left of the network visual display, as opposed to the bottom-right. In order to control for such spurious effects, we implemented the four treatments in each of four sessions keeping constant the networks structure, while changing in each session the spatial position of the nodes, as detailed in Figure $5 .{ }^{18}$ The same four sets of signals were used in each of the four sessions. Therefore, by implementing the four network nodes with all possible labels and visual locations, while keeping signals constant, we were able to control for any possible confounding effects and cleanly identify the causal effects of network structure. In what follows, unless otherwise stated, we will refer to the four network nodes using the labels of session 1 . This means that nodes from sessions 2 to 4 are implicitly relabeled so that they are topologically the same as in session 1 .

Figure 5: Network structure, by session
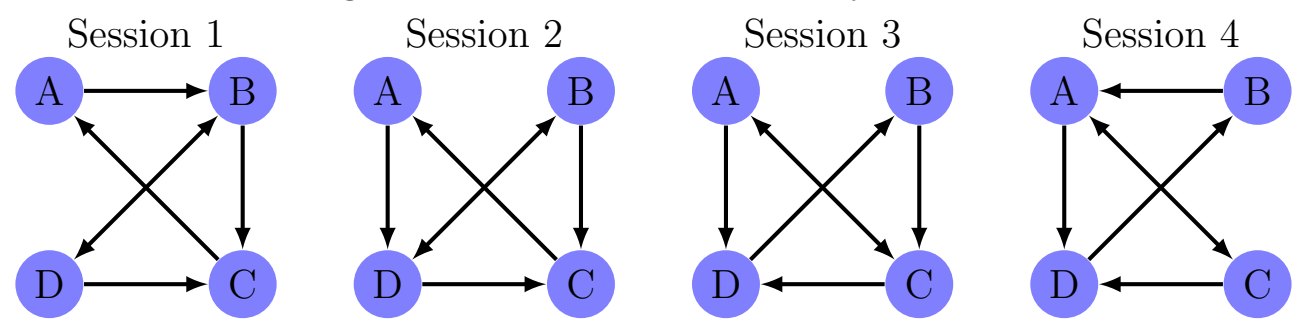

\subsection{Hypotheses}

Consider the network structure in Figure 4. As detailed in Table 1, there exists a set of strategies that allows each of the four network members to find out $\bar{x}$ in just four rounds. Indeed, there exist several possible combinations of strategies that result in correct beliefs. With such optimal strategies, the four agents have equal social influence weights in consensus beliefs, i.e. $w=w^{*}=[0.25,0.25,0.25,0.25]$.

Let us now consider the predictions for the GBR updating rule described in equations (1) and (3). Figure 6 shows how the social influence weights for each of the four network nodes change as a function of $\rho$. When $\rho=0$, agents $B$ and $D$ are the most influential. This, loosely speaking, reflects the fact that both $B$ and $D$ have an outdegree of 2, while $A$ and $C$ have an outdegree of 1: the agents who are listened to by more other agents are

\footnotetext{
${ }^{18}$ This means that, for instance, the node that has label B and upper-right position in session 1, has then label D and bottom-left position in session 2, label C and bottom-right position in session 3, label A and upper-left position in session 4, respectively.
} 
Table 1: Optimal strategies for each network position, by round

\begin{tabular}{ccccc}
\hline \hline Round & $A$ & $B$ & $C$ & $D$ \\
\hline 1 & $x_{A}$ & $x_{B}$ & $x_{C}$ & $x_{D}$ \\
2 & $\frac{x_{A}+y_{C}^{1}}{2}$ & $\frac{x_{B}+y_{A}^{1}+y_{D}^{1}}{3}$ & $\frac{x_{C}+y_{B}^{1}+y_{D}^{1}}{3}$ & $\frac{x_{D}+y_{B}^{1}}{2}$ \\
3 & $\frac{x_{A}+3 y_{C}^{2}}{4}$ & $\frac{x_{B}+2 y_{A}^{2}+y_{D}^{1}}{4}$ & $\frac{x_{C}+3 y_{B}^{2}}{4}$ & $\frac{x_{D}+3 y_{B}^{2}-y_{D}^{1}}{3}$ \\
4 & $y_{A}^{t-1}$ & $y_{B}^{t-1}$ & $y_{C}^{t-1}$ & $\frac{x_{D}+4 y_{B}^{3}-y_{D}^{1}}{4}$ \\
$\geq 5$ & $y_{A}^{t-1}$ & $y_{B}^{t-1}$ & $y_{C}^{t-1}$ & $y_{D}^{t-1}$ \\
\hline
\end{tabular}

Note: agents' positions in column headings refer to the network structure displayed in

Figure 4.

the most influential. ${ }^{19}$ Also note that $A$ is relatively more influential than $C$. This reflects indirect social influence, as $A$ communicates to $B$, who is one of the two most influential subjects, while $C$ communicates to $A$. For $\rho>0$, the pattern of social influence weights also reflects agents' indegree. In particular, as $\rho$ increases, $B$ becomes progressively more influential, while the weights of the other three agents decrease.

Figure 6: Social influence weights as a function of $\rho$

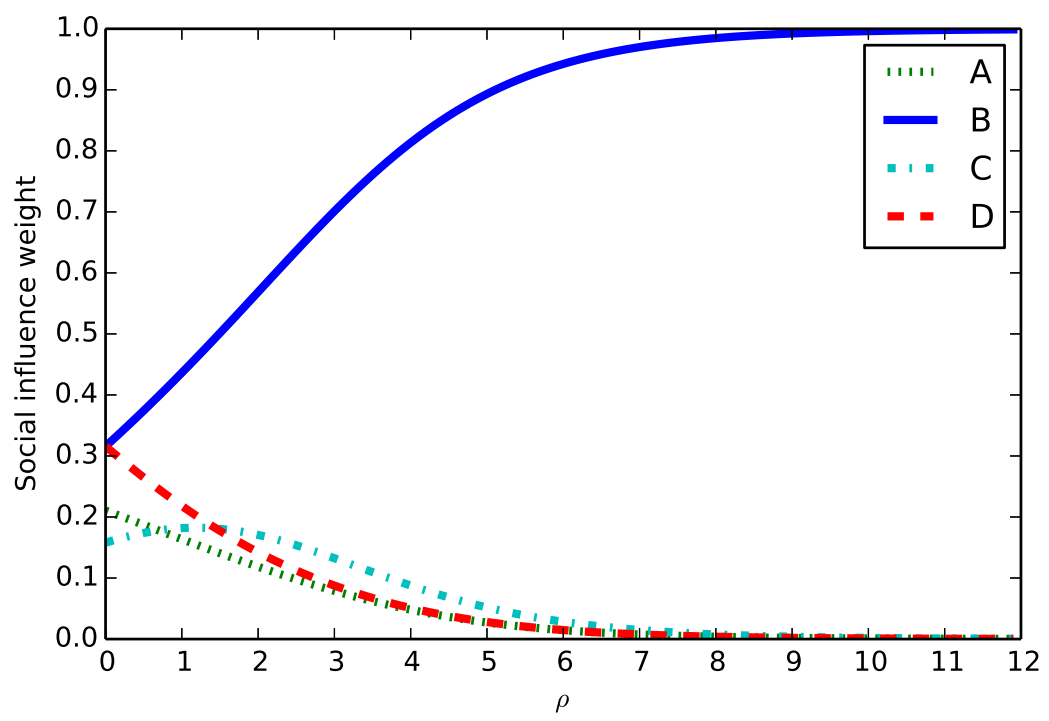

To summarize, Table 2 compares point predictions of social influence weights for $\rho=0, \rho=1$ and $\rho \rightarrow \infty$. When $\rho=0$, as in DeMarzo et al.

\footnotetext{
${ }^{19}$ Note that $\mathrm{B}$ and $\mathrm{D}$ have exactly the same weight since they communicate to the same individuals ( $C$ and each other) and, while the link from $A$ to $B$ implies that the latter is placing a lower weight $\left(\frac{1}{3}\right.$ rather than $\left.\frac{1}{2}\right)$ on her own belief, she is hence placing lower weight also on the information coming from $D$.
} 
(2003), $B$ and $D$ have equal social influence weights, similarly to the case of Bayesian updating. When $\rho=1$, so that agents update their beliefs using weights that are proportional to indegree, $B$ is the most influential agent. Finally, when $\rho \rightarrow \infty$, consensus beliefs tend to agent $B$ 's initial opinion.

Table 2: Predictions for social influence weights for different values of $\rho$

\begin{tabular}{lcccc}
\hline \hline & $w_{A}$ & $w_{B}$ & $w_{C}$ & $w_{D}$ \\
\hline$\rho=0$ & 0.21 & 0.32 & 0.16 & 0.32 \\
$\rho=1$ & 0.18 & 0.39 & 0.18 & 0.26 \\
$\rho \rightarrow \infty$ & 0.00 & 1.00 & 0.00 & 0.00 \\
\hline Note: the predictions refer to the updating rule described in equations (1) and (3) for \\
the network structure displayed in Figure 4.
\end{tabular}

The pattern described in Figure 6 and Table 2 provides the predictions tested in the experiment. We start by testing the hypothesis that agents optimally update their beliefs. Empirically, the null hypothesis is that all nodes have equal weights in consensus beliefs:

$$
H_{0}: w_{A}=w_{B}=w_{C}=w_{D}=0.25
$$

Note that this is a general test of Bayesian updating versus an unspecified alternative. In order to test against the specific alternative of the generalized boundedly rational updating rule, we focus on pair-wise differences between individual weights. More specifically, as shown in Figure 6, the updating rule predicts that, for any $\rho \geq 0$, an agent in node $B$ is more influential than in either $A$ or $C$. Conversely, for all other pair-wise comparisons between nodes, the sign of the difference between weights is not independent of $\rho$. Therefore, the relevant one-sided hypotheses can be stated as follows:

$$
\begin{aligned}
& H_{0}: w_{B} \leq w_{A} \text { vs } H_{1}: w_{B}>w_{A} \\
& H_{0}: w_{B} \leq w_{C} \text { vs } H_{1}: w_{B}>w_{C}
\end{aligned}
$$

Finally, we test our key hypothesis about the effect of indegree on social influence. For $\rho=0$, similarly to the case of Bayesian updating, the GBR updating rule predicts $w_{B}=w_{D}$. On the other hand, for $\rho>0$, the GBR rule predicts $w_{B}>w_{D}$ (see Figure 6). We can thus test the effect of indegree on social influence by comparing the social influence weights of agents $B$ and $D$ :

$$
H_{0}: w_{B} \leq w_{D} \text { vs } H_{1}: w_{B}>w_{D}
$$




\subsection{Procedures}

The experiment was conducted in the Experimental Economics Lab of the University of Milan Bicocca between January and March 2013, with 24 subjects participating in each of the four sessions (96 in total). Subjects were undergraduate students, recruited by email through an online system. The experiment was ran using z-Tree (Fischbacher, 2007). Subjects on average earned 7.7 euro (earnings were strictly positive for 63 participants out of 96), plus the show-up fee of 5 euro, for sessions lasting approximately 80 minutes, including time for instructions, control questions and payments.

Each session consisted of four eight-round phases. Subjects were informed that signals and network positions would be randomly determined at the beginning of each phase, while the composition of the groups would remain unchanged throughout the session. Subjects were only informed that the signals would be integer numbers randomly drawn by the system.

All the experimental instructions, reported in Appendix B, were provided to the participants in written form, and also read aloud at the beginning of the session. Individuals were then asked to answer some control questions. Each participant had the possibility to take notes and make calculations on paper, and also to use an on-screen calculator. Moreover, in each round, the screen reported all the information available (own past guesses and past guesses of neighbors since the beginning of the phase), in order to guarantee perfect recall.

The instructions explicitly suggested that, had an individual known with certainty a subset of the signals for her group, her optimal strategy was to report their average. This, together with the fact that individuals had to target the average of four specific numbers (rather than the mean of an underlying distribution of signals) helped us to minimize mistakes caused by inappropriate statistical inference, thus ensuring that individuals could focus on the process of information aggregation.

\section{Results}

In each of the four sessions, the experimental task was implemented by 24 subjects over 8 rounds in four different phases (32 rounds in total), resulting in 384 observations for each round (24 subjects $\times 4$ sessions $\times 4$ phases) and 3072 observations in total. Overall, although there was substantial heterogeneity at individual and group level, subjects generally showed to have clearly understood the experimental task. In the first round of each phase, 94.2 per cent of the subjects truthfully reported their own signal, while 96 per cent of the subjects reported a number within 10 units from their own signal. In the final round of each phase, 24 per cent of the subjects correctly 
guessed the average of the four signals within their group and, accounting for rounding errors, 55.5 per cent of the subjects reported beliefs within 10 units from the average of the four signals.

\subsection{Tests of Hypotheses}

In order to test hypotheses about the social influence weights of agents in different network positions, we specify each agent's final belief as a linear combination of the initial signals of the four agents in her group:

$$
y_{i}^{T}=\mu+w_{A} x_{i, A}+w_{B} x_{i, B}+w_{C} x_{i, C}+w_{D} x_{i, D}+\varepsilon_{i}
$$

where $y_{i}^{T}$ is agent $i$ 's belief in the last round (round 8) of each phase, $x_{i, j}$ is the signal observed by agent $j$ in $i$ 's group, $\mu$ is a constant, $w_{j}$ is the social influence weight of agent $j$, and $\varepsilon_{i}$ is an idiosyncratic error term. Equation (11) is estimated by OLS, under the constraint $\sum_{j} w_{j}=1$. The set of regressors also includes sets of dummy variables for sessions and phases. In order to take into account the dependence of observations belonging to the same group within each session, standard errors are clustered by 24 independent groups (there are 6 independent groups in each of the four sessions).

Table 3 presents the results. Since we are focusing on the final observation from each of the four phases, the overall sample includes 384 individual observations. Column (1) reports estimates of social influence weights in absolute terms, as in equation (11). The weights generally differ from 0.25 , with a pattern that is qualitatively consistent with the predictions of the GBR rule: social influence is highest for node $B$ and lowest for node $C$. The hypothesis that all nodes have equal weight $\left(w_{A}=w_{B}=w_{C}=w_{D}=0.25\right)$, as predicted under Bayesian updating, is strongly rejected by the data $(p<0.01)$.

Result 1: Bayesian updating is rejected by the data.

Focusing on pair-wise differences between weights (hypotheses H2-H3), $w_{B}$ is higher than $w_{C}$, consistent with the predictions of the generalized boundedly rational rule, and the difference $(+0.080)$ is strongly significant $(p<0.01)$. Similarly, $w_{B}$ is higher than $w_{A}$, although the difference $(+0.026)$ is not significant $(p<0.25)$.

Result 2: Pair-wise differences between social influence weights are consistent with the GBR updating rule.

Turning to our key hypothesis about the role of indegree, we find that, as predicted by the GBR rule in this network structure for $\rho>0, w_{B}(0.294)$ is higher than $w_{D}(0.224)$ and the difference is large and strongly significant $(p<0.01)$. This leads us to reject the null hypothesis that $\rho=0$. 
Table 3: Estimated social influence weights, overall

\begin{tabular}{lcc}
\hline \hline & $(1)$ & $(2)$ \\
& Absolute weights & Relative weights \\
\hline Signal A & $0.268^{* * *}$ & 0.018 \\
& $(0.031)$ & $(0.031)$ \\
Signal B & $0.294^{* * *}$ & $0.044^{* * *}$ \\
& $(0.013)$ & $(0.013)$ \\
Signal C & $0.214^{* * *}$ & $-0.036^{* *}$ \\
& $(0.017)$ & $(0.017)$ \\
Signal D & $0.224^{* * *}$ & -0.026 \\
& $(0.023)$ & $(0.023)$ \\
\hline Number of observations & 384 & 384 \\
\hline Note: the table reports OLS estimates of social influence weights associated to the node \\
indicated by the row heading. The weights are expressed in absolute terms (column 1) \\
and as a difference from 0.25 (column 2), respectively. Dependent variable: individual \\
beliefs in final round. All specifications include full sets of session and phase dummies. \\
Standard errors clustered at group level reported in brackets. ${ }^{* * *}$ p $<0.01,{ }^{* *} \mathrm{p}<0.05$, \\
${ }^{*} \mathrm{p}<0.10$.
\end{tabular}

Result 3: The social influence of an individual is positively affected by the number of individuals she listens to.

This is an important finding, as it provides causal evidence that subjects do not place equal weights on all their neighbors, but take into account their neighbors' indegree when aggregating the information they receive from them. As a result, ceteris paribus, subjects with higher indegree ultimately have higher social influence.

In order to shed light on these findings, column (2) reports differences of social influence weights with respect to 0.25 , obtained by expressing individual final-round beliefs as deviations from the average of the four group signals. The results indicate that $w_{B}$ is significantly higher than $0.25(p<0.01)$, while $w_{C}$ is significantly lower than $0.25(p<0.02)$. On the other hand, $w_{A}$ and $w_{D}$ are not significantly different from $0.25(p<0.29$ and $p<0.13$, respectively, for the corresponding one-sided hypothesis). The findings for the social influence weights of nodes $B$ and $D$ provide further evidence against a simple updating rule where all neighbors receive equal weight.

\subsection{Robustness}

In order to assess the robustness of the results to the possible effects of outliers, Table 4 reports estimates of (relative) social influence weights obtained by eliminating from the sample the groups containing the $1 \%, 5 \%$, 
or $10 \%$ most extreme observations. Potential outliers are identified by considering, for each group member, the difference between the reported beliefs and the ones predicted under Bayesian updating. This results in a restricted sample size of 380, 364 and 344 observations, respectively. In all cases, point estimates and test results are virtually unchanged relative to the overall sample. The hypothesis that all nodes have equal weight $\left(w_{A}=w_{B}=w_{C}=w_{D}=0.25\right)$ is strongly rejected by the data $(p<0.01)$. The hypothesis that $w_{B}=w_{D}$ is also strongly rejected in all cases. Indeed, by eliminating potential outliers, the estimated weights are even more closely consistent with the theoretical predictions of the GBR updating rule. In column (3), for example, where the 10 per cent of the groups reporting the most extreme deviations from optimal predictions are excluded, the estimated relative weights for the four nodes are $0.005,0.053,-0.045$ and -0.012 , respectively. In all cases, $w_{B}\left(w_{C}\right)$ is significantly higher (lower) than 0.25 .

Table 4: Social influence (relative weights), robustness

\begin{tabular}{lccc}
\hline \hline & $(1)$ & $(2)$ & $(3)$ \\
& $1 \%$ & $5 \%$ & $10 \%$ \\
\hline Signal A & 0.013 & 0.019 & 0.005 \\
Signal B & $(0.031)$ & $(0.032)$ & $(0.027)$ \\
& $0.049^{* * *}$ & $0.051^{* * *}$ & $0.053^{* * *}$ \\
Signal C & $(0.014)$ & $(0.015)$ & $(0.014)$ \\
& $-0.037^{* *}$ & $-0.045^{* *}$ & $-0.045^{* * *}$ \\
Signal D & $(0.017)$ & $(0.018)$ & $(0.017)$ \\
& -0.026 & -0.024 & -0.012 \\
Number of observations & $(0.023)$ & $(0.023)$ & $(0.017)$ \\
\hline
\end{tabular}

Note: the figures reported are estimates of social influence weights, as a difference from 0.25 , associated to the subject in the position indicated by the row heading. Dependent variable: individual beliefs in final round. All specifications include full sets of session and phase dummies. Standard errors clustered at group level reported in brackets. ${ }^{* * *} \mathrm{p}<0.01,{ }^{* *} \mathrm{p}<0.05,{ }^{*} \mathrm{p}<0.10$. Columns (1) to (3): sample restricted by eliminating groups with most extreme deviations from optimal beliefs $(1 \%, 5 \%, 10 \%$, respectively).

It should be observed that, although the variance of beliefs held by the four group members falls steadily over successive rounds in all groups, disagreement persists in many cases, so that beliefs do not converge to a consensus in all cases. In order to assess the potential effects of non-convergence, Table 5 presents estimates of relative social influence weights by individual network position. Focusing on nodes $B$ and $D$ (columns 2 and 4 ), estimated social influence weights are qualitatively unchanged with respect to the re- 
sults in Table 3: the relative weight of $B$ is positive and significant, while it is negative and significant for $C$. Agent $D$ has a negative relative weight in the final beliefs of agent $A$. Finally, relative social influence weights are not different from zero for $C$.

Table 5: Social influence (relative weights), by node

\begin{tabular}{lcccc}
\hline \hline & $(1)$ & $(2)$ & $(3)$ & $(4)$ \\
& Node A & Node B & Node C & Node D \\
\hline Signal A & 0.057 & 0.015 & 0.007 & -0.009 \\
& $(0.047)$ & $(0.037)$ & $(0.040)$ & $(0.066)$ \\
Signal B & 0.038 & $0.057^{* *}$ & 0.015 & $0.065^{* *}$ \\
& $(0.038)$ & $(0.024)$ & $(0.023)$ & $(0.026)$ \\
Signal C & 0.013 & $-0.070^{* *}$ & 0.024 & $-0.110^{* * *}$ \\
& $(0.033)$ & $(0.027)$ & $(0.022)$ & $(0.035)$ \\
Signal D & $-0.109^{* *}$ & -0.003 & -0.045 & 0.053 \\
& $(0.041)$ & $(0.025)$ & $(0.031)$ & $(0.048)$ \\
\hline Number of observations & 96 & 96 & 96 & 96
\end{tabular}

Note: the figures reported are estimates of the social influence weights, as a difference from 0.25 , associated to the subject in the position indicated by the row heading.

Dependent variable: individual beliefs in final round. All specifications include full sets of session and phase dummies. Standard errors clustered at group level reported in brackets. ${ }^{* * *} \mathrm{p}<0.01,{ }^{* *} \mathrm{p}<0.05,{ }^{*} \mathrm{p}<0.10$.

The results in Table 5 also indicate that agents at all nodes place higher than average weight on their own signal (positive estimates along the main diagonal). This raises the question of whether indegree is relevant beyond the role of own signal. That is, whether the test results for $\mathrm{H} 4$ are driven by agents at node $B$ placing higher weight on their own signal than agents at node $D$. We thus re-estimated equation (10) by allowing for the coefficients to differ, at each node, between the two cases of own signal and others' signal. The estimated absolute social influence weights of $B$ and $D$ are 0.316 and 0.258 , respectively, for own signal, and their difference is not statistically significant $(p<0.251)$. For others' signal, the estimated weights of $B$ and $D$ are 0.272 and 0.196 , respectively, and their difference is instead strongly significant $(p<0.001)$. This indicates that indegree matters for the importance attributed to others', rather than own, opinions.

Overall, these results indicate that the effects of network structure on social influence reported in Section 4.1 are qualitatively and quantitatively robust to the potential effects of outliers. In addition, they are qualitatively unaffected by the possible non-convergence of beliefs within individual groups. 


\subsection{Further Evidence}

The experimental data also allow us to investigate the mechanisms underlying the observed treatment effects on social influence weights, by looking at how agents at specific nodes aggregate the information they receive in each round. At individual level, there can be two possible, not mutually exclusive, mechanisms explaining differences in social influence between nodes $B$ and $D$. The first and most intuitive mechanism is that $C$ may place a higher weight on the opinion of $B$ than on that of $D$, because of $B$ 's higher indegree. The second is that, for the same reason, $D$ may be influenced by $B$ relatively more than $B$ is influenced by $D$.

Table 6 sheds light on this issue by presenting estimates of node-specific (absolute) weights based on all updating rounds. Looking at the estimates for node $C$ (column 3 ), the weight of $B(0.435)$ is substantially higher than the one of $D(0.163)$, and the difference is strongly statistically significant $(p<$ 0.01). This provides support to the first of the two mechanisms described above. The comparison of the weights given to each other by $B$ and $D$ is non-trivial, since their respective indegrees are different, as they form their beliefs on the basis of different numbers of neighbors. However, $D$ appears to substantially under-weigh the information coming from $B(0.104)$, whereas $B$ does not substantially under-weigh the information received from $D(0.325)$. The overall effect of indegree on social influence is therefore mainly explained by the way in which information is processed by $C$ : node $B$, whose indegree is twice the indegree of $D$, receives a weight that is more than twice as large as the weight for node $D$.

Since the hypothesis that $\rho=0$ is strongly rejected by the data, it is interesting to ask what value of $\rho$ provides the best fit for the experimental data. We simulated the generalized updating rule, searching for the value of $\rho$ that minimizes the sum of squared deviations, over all individuals, between observed (experimental) and simulated final-round beliefs:

$$
\hat{\rho}=\underset{\rho}{\arg \min } \sum_{g=1}^{96} \sum_{k=1}^{4}\left(y_{g, k, T}-\bar{y}_{k, T}^{\rho}\right)^{2},
$$

where $y_{g, k, T}$ is the belief of an individual with role $k$ in group $g$ in the final round, and $\bar{y}_{k, T}^{\rho}$ is the corresponding theoretical prediction. This produces an estimate of $\hat{\rho}=0.30$. Interestingly, this is higher than the efficient $\rho^{*}=$ 0.04 (see Figure 3). This indicates that agents place higher weight on those neighbors who themselves listen to more peers, as they should, but do so to a greater extent than would be optimal. 
Table 6: Neighbors' absolute weights in current beliefs, by node

\begin{tabular}{lcccc}
\hline \hline & $(1)$ & $(2)$ & $(3)$ & $(4)$ \\
& Node A & Node B & Node C & Node D \\
\hline Lagged belief, node A & $0.530^{* *}$ & 0.000 & & \\
& $(0.011)$ & $(0.000)$ & & \\
Lagged belief, node B & & $0.675^{* *}$ & $0.435^{* *}$ & $0.104^{* *}$ \\
& & $(0.070)$ & $(0.087)$ & $(0.018)$ \\
Lagged belief, node C & $0.470^{* *}$ & & $0.402^{* *}$ & \\
& $(0.011)$ & & $(0.087)$ & \\
Lagged belief, node D & & $0.325^{* *}$ & $0.163^{* *}$ & $0.896^{* *}$ \\
& & $(0.070)$ & $(0.049)$ & $(0.018)$ \\
\hline Number of observations & 672 & 672 & 672 & 672 \\
\hline Note: the figures reported are estimates of neighbors' weights, based on all updating \\
rounds. Dependent variable: current belief of agent at the node reported in column \\
heading, rounds 2-8. All specifications also include full sets of session and phase \\
dummies. Standard errors clustered at independent group level reported in brackets. \\
${ }^{* * *}$ p $<0.01,{ }^{* *}$ p $<0.05,{ }^{*}$ p $<0.10$. \\
$5 \quad$ Conclusions
\end{tabular}

Although the mathematical concept of digraph, i.e., a network based on directed relations, was already central in the pioneering works of French (1956) and Harary (1959), empirical studies of information diffusion in social networks have generally not focused explicitly on the respective roles played by indegree and outdegree. This may reflect the fact that, while asymmetric information flows are the norm in opinion formation, most available network data sets, such as those describing friendship relations on online social networks, co-authorships of academic authors, or traffic flows, generally describe undirected networks. Recently, however, increasing attention has been given, both theoretically and empirically, to information flows in directed networks (e.g. Baños et al., 2013, Gleeson et al., 2014).

In this paper, we investigated the properties of the boundedly rational model of opinion formation in directed social networks proposed by Corazzini et al. (2012). In this model, that provides a simple generalization of the updating rule in DeMarzo et al. (2003), agents aggregate the information they receive from their neighbors' by using weights that depend positively on their neighbors' indegree. Intuitively, when opinions are updated, relatively more importance is attributed to the opinions of more informed individuals. Although simple, this generalization introduces an important novelty in that subjects update their opinion by taking into account not only their neighbors' opinions but also the features of the structure of the communication network. 
At the theoretical level, our results indicate that, in balanced networks, placing higher weight on neighbors with higher indegree is generally less efficient than placing equal weights on all neighbors. On the other hand, in unbalanced networks, it can be efficient to place higher weight on neighbors with higher indegree. Indeed, there exist unbalanced networks in which the optimal weight of indegree is arbitrarily high. At the empirical level, our experimental results provide clean evidence of a positive causal effect of indegree on social influence. Both Bayesian updating and naive updating by placing equal weights on all neighbors are rejected against the alternative of an updating rule in which the weight placed on an agent's opinion is positively related to the number of individuals she listens to. Indeed, the sensitivity of updating weights to neighbors' indegree is found to be higher than would be efficient.

One possible interpretation of our findings is that agents are aware that, in the setting considered, placing a higher weight on neighbors with higher indegree is efficient. However, in their attempt to aggregate information efficiently while using a simple updating rule, they end up placing excessive weight on neighbors with high indegree. A second possible explanation is that, irrespective of any efficiency motivation, agents tend to attribute some form of authority to peers whom they perceive as better informed, and this leads them to place a higher weight on the information received from them. A further possible interpretation of our results is that the weights of the updating rule could be state-dependent. In the framework by Hegselmann and Krause (2002), for example, updating weights depend negatively on the distance between opinions. Since the beliefs of high-indegree agents are, on average, less extreme than those of low-indegree agents, they can be expected to be more similar, on average, to the beliefs of the listening agents. In this perspective, our results could be interpreted as reflecting features of beliefs, so that network structure, and more specifically indegree, would play a role only indirectly.

To sum up, our analysis provides causal evidence of an indegree effect that is at odds with the updating mechanisms most commonly adopted in the literature on opinion dynamics. When forming their opinion, agents do not place equal weights on all their neighbors, but use weights that are positively related to their neighbors' indegree. As a result, ceteris paribus, subjects with higher indegree have higher social influence. This is an important finding, as it indicates that, despite their inability to fully account for the structure of their communication network, agents are able to exploit the information about its local properties. Further research should contribute to an understanding of the mechanisms explaining the effect of indegree on social influence. 


\section{Appendix A - Theorems and Proofs}

In the following two theorems, it is assumed that the network $\mathcal{G}$ is strongly connected and that it contains no direct link between $\bar{i}$ and $\bar{j}$. The modified network will be denoted as $\mathcal{G}^{\prime}$. The updating weights adopted in the original and modified networks will be denoted as $\pi$ and $\pi^{\prime}$, the listening sets as $S(i)$ and $S(i)^{\prime}$, the vectors of updating weights as $w$ and $w^{\prime}$, respectively. $\Delta=w^{\prime}-w$ represents the vector of changes of social influence weights and $\delta=\frac{\Delta}{w}$ the vector of relative changes.

Theorem A1 Let $\bar{i}$ and $\bar{j}$ be two nodes in $\mathcal{G}$. Under the GBR updating rule, provided $\rho$ is not too large, adding a link from $\bar{i}$ to $\bar{j}$ increases the social influence of $\bar{i}$.

Intuitively, Theorem A1 states that, except for extreme values of $\rho$ such that the indegree effect on the receiving node may dominate, social influence is increasing in outdegree. In order to prove Theorem A1, we first prove the following.

Lemma A1 There exists a $\rho^{*}$ such that, under the GBR rule with $\rho \in\left[0, \rho^{*}\right)$, $\delta_{\bar{i}} \geq \delta_{i}$ for all $i$.

Proof of Lemma A1. Equation (8) allows us to express the social influence of an agent as a linear combination of the social influence weights of the agents she talks to, so that, analogously:

$$
\delta_{i}=\frac{w_{i}^{\prime}-w_{i}}{w_{i}^{\prime}}=\sum_{j \in S^{-1}(i)^{\prime}} \frac{\pi_{j i}^{\prime} w_{j}^{\prime}-\pi_{j i} w_{j}}{w_{i}} .
$$

Let us define $\hat{\delta}_{i}$ (the component of $\delta_{i}$ imputed to a change in the listeners' influence) as:

$$
\hat{\delta}_{i}=\sum_{j \in S^{-1}(i)} \frac{\pi_{j i}\left(w_{j}^{\prime}-w_{j}\right)}{w_{i}}=\sum_{j \in S^{-1}(i)} \frac{\pi_{j i} w_{j} \delta_{j}}{w_{i}}
$$

We can observe that:

- $\delta_{\bar{i}}>\hat{\delta}_{\bar{i}}$, since $S^{-1}(\bar{i})^{\prime}=S^{-1}(\bar{i}) \cup\{\bar{j}\}$, while $\pi_{j \bar{i}}=\pi_{j \bar{i}}^{\prime}$ for all $j \neq \bar{j}$,

- $\delta_{\bar{j}}<\hat{\delta}_{\bar{j}}$ as long as $\rho$ is smaller than some $\rho^{*}$, since $\pi_{\bar{j} \bar{j}}<\pi_{\bar{j} \bar{j}}^{\prime}$ for $\rho=0$ and the updating weights are continuous in $\rho$; in particular, $\rho^{*}>1$ if $d_{\bar{i}} \geq d_{h}$ for all others $h \in S(\bar{j})$,

- $\delta_{i}<\hat{\delta}_{i}$ for any other $i \in S(\bar{j})$, since $\pi_{\bar{j} i}^{-}<\pi_{\bar{j} i}^{\prime}$, 
- $\delta_{i}=\hat{\delta}_{i}$ for any $i \notin S(\bar{j})^{\prime}$.

Now, assume Lemma A1 is false. That is, there exists $\bar{h} \neq \bar{i}$ such that $\delta_{\bar{h}}>\delta_{\bar{i}}$. Let us assume, without loss of generality, that $\delta_{\bar{h}} \geq \delta_{i}$ for all $i$. We can then write:

$$
\begin{aligned}
\delta_{\bar{h}} & \leq \hat{\delta}_{\bar{h}}=\sum_{j \in S^{-1}(\bar{h})} \frac{\pi_{j \bar{h}} w_{j} \delta_{j}}{w_{\bar{h}}} \\
& \leq \sum_{j \in S^{-1}(\bar{h})} \frac{\pi_{j \bar{h}} w_{j} \delta_{\bar{h}}}{w_{\bar{h}}}=\frac{\delta_{\bar{h}}}{w_{\bar{h}}} \sum_{j \in S^{-1}(\bar{h})} \pi_{j \bar{h}} w_{j}=\delta_{\bar{h}} \frac{w_{\bar{h}}}{w_{\bar{h}}}=\delta_{\bar{h}} .
\end{aligned}
$$

We can see that both inequalities must be binding. The first implies that $\bar{h} \notin S(\bar{j})^{\prime}$, and hence $\bar{h} \notin S(\bar{j})$; the second implies that $\delta_{j}=\delta_{\bar{h}}$ for all $j \in S^{-1}(\bar{h})$. By applying the same process recursively to any such $j$, and exploiting the strong connectedness of the network, it follows that $\delta_{\bar{i}}=\delta_{\bar{h}}$, which contradicts the initial assumption. ${ }^{20}$

Proof of Theorem A1. The sum of social influence weights is by definition 1 in any network, so that the weighted sum of percentage changes must be 0 :

$$
\sum_{i=1}^{n} w_{i}=1=\sum_{i=1}^{n} w_{i}^{\prime} \Longrightarrow \sum_{i=1}^{n} w_{i} \delta_{i}=0
$$

(with all weights $w_{i}$ strictly positive). Since $\hat{\delta}_{i}$ is a linear combination of $\delta_{j}$ for different $j$, if we had $\delta_{j}=0$ for all $j$, then we would have $\hat{\delta}_{i}=0$ for all $i$. Instead, we know that $\delta_{j}<\hat{\delta}_{j}$ for some $j$. So the maximum $\delta_{i}$, which is guaranteed by Lemma A1 to be $\delta_{\bar{i}}$, must be strictly positive.

Theorem A2 Let $\bar{i}$ and $\bar{j}$ be two nodes in $\mathcal{G}$. Under the GBR updating rule:

(i) for $\rho=0$, as long as there is no path from $\bar{j}$ to $\bar{i}$ shorter than $d_{\bar{j}}$, adding a link from $\bar{i}$ to $\bar{j}$ decreases the social influence of $\bar{j}$;

(ii) for $\rho>0$ sufficiently large, as long as $d_{\bar{i}} \leq d_{\bar{j}}$, adding a link from $\bar{i}$ to $\bar{j}$ increases the social influence of $\bar{j}$.

Intuitively, Theorem A2 states that for $\rho=0$ a higher indegree implies lower self-importance thus decreasing social influence, while for $\rho>0$ a higher indegree implies receiving a higher weight, thus increasing social influence. Hence, for sufficiently large values of $\rho$, the overall effect of indegree on social

\footnotetext{
${ }^{20}$ If the chosen path from $\bar{h}$ to $\bar{i}$ passes through $S_{1}^{-1}(\bar{j})$, the contradiction will arise even before reaching $\bar{i}$.
} 
influence is positive. The additional assumptions cope with the possible indirect effects due to the change of influence of $\bar{i}$.

Proof. For point ( $i$ ), notice that the influence of any node $j$ can be decomposed as $w_{j}=\frac{\sum_{k=0}^{\infty} \pi_{j j}^{k} \cdot \sigma_{j}}{n}=\frac{\lambda_{j} \cdot \sigma_{j}}{n}$, where $\lambda_{j}=\frac{1}{\left(1-\pi_{j j}\right)}=\frac{1}{\left(1-\frac{1}{d}\right)_{j}}$ describes the "flow of opinion $j$ " which is transferred to the rest of the network during the process of opinion formation, and $\sigma_{j}$ describes the effect of the diffusion in the network (including subsequent passes through $j$ ). When the indegree of $\bar{j}$ increases from $d_{\bar{j}}$ to $d_{\bar{j}}+1, \lambda_{j}$ decreases by a coefficient $\frac{1}{d_{\bar{j}}\left(d_{\bar{j}}-1\right)}$. On the other hand, for a recursive reasoning analogous to the one used in the proof of Theorem A1, any increase of influence through $\sigma_{\bar{j}}$ ultimately depends on an increase of $w_{\bar{i}}$, which is however bounded above by $\pi_{\bar{j} \bar{i}}^{\prime} w_{\bar{j}}^{\prime}=\frac{1}{d_{\bar{j}}+1} w_{\bar{j}}^{\prime}$. In turn, the benefit accruing to $\bar{j}$ in terms of influence is bounded above by $\frac{1}{d_{\bar{j}}} w_{\bar{i}}^{\delta}$ : since the shortest path between $\bar{j}$ and $\bar{i}$ is long at least $d_{\bar{j}}$, there are at least $d_{\bar{j}}$ classes of nodes (those at distance $0,1, \ldots, d_{\bar{j}}-1$ from $\bar{i}$ on a path from $\bar{j}$ to $\bar{i}$ ) each benefiting from the increase of $w_{\bar{i}}$ more than $\bar{j}$. So we have

$$
\Delta_{\bar{j}} \leq-\frac{1}{d_{\bar{j}}\left(d_{\bar{j}}-1\right)} w_{\bar{j}}^{\prime}+\frac{1}{d_{\bar{j}}} \frac{1}{d_{\bar{j}}+1} w_{\bar{j}}^{\prime} \Longrightarrow \Delta_{\bar{j}}<0 .
$$

For point (ii), recall from equation 5 that when $\rho \rightarrow \infty$ each agent will only listen to the agent(s) with highest indegree in her listening set. Given any network $G$, let $\mathcal{C}(G)$ be the network obtained by removing from $G$ each link from $i$ to $j$ with $d_{i}<d_{j}$ (notice that if $G$ is strongly connected $\mathcal{C}(G)$ will be connected, but not necessarily strongly). The social influence ${ }^{21}$ of a node in $G$ under $\rho \rightarrow \infty$ is equal to the social influence of that node in $\mathcal{C}(G)$ under $\rho=0$. Now assume that $\mathcal{C}\left(\mathcal{G}^{\prime}\right)$ contains the link from $\bar{i}$ to $\bar{j}$ : then the social influence of $\bar{i}$ under $\rho=0$ in $\mathcal{C}\left(\mathcal{G}^{\prime}\right)$ is larger than in $\mathcal{C}(\mathcal{G})$, so that, for sufficiently large values of $\rho$ this is also true in $\mathcal{G}^{\prime}$ compared to $\mathcal{G}$. If instead $\mathcal{C}\left(\mathcal{G}^{\prime}\right)$ does not contain such link, the assumption $d_{\bar{i}} \leq d_{\bar{j}}$ guarantees that the influence of node $\bar{j}$ tends to 0 both in $\mathcal{G}$ and in $\mathcal{G}^{\prime}$. Let $\hat{i}$ the node with maximum indegree found in $S(\bar{j}): \pi_{\bar{j} \bar{i}}$ converges in $\mathcal{G}^{\prime}$ to 0 as $w_{\bar{i}} \cdot\left(\frac{d_{\bar{i}}+1}{d_{\hat{i}}}\right)^{\rho}$ rather than as $w_{\bar{i}} \cdot\left(\frac{d_{\bar{i}}}{d_{\hat{i}}}\right)^{\rho}$, while $\pi_{i j}$ is asymptotically unchanged for any other $i, j$. Hence for sufficiently large values of $\rho$ the social influence of $\bar{i}$ increases.

${ }^{21}$ The social influence of a node has been defined only in the case in which opinions converge to a consensus. Here we implicitly use a more general definition obtained by simply replacing $y^{\infty}$ with $\frac{\sum_{i} y_{i}^{\infty}}{n}$ in equation 7 . 
Proof of Theorem 1. Consider a strongly connected network $\mathcal{G}$ : its adjacency matrix $M_{\mathcal{G}}=\left\{q_{i j}\right\}_{i, j \leq n}$ is necessarily irreducible. ${ }^{22}$ Perfect and Mirsky (1965) have shown ${ }^{23}$ that then there exists another matrix $P_{\mathcal{G}}$, with coefficients $p_{i j}$ such that

- $P_{\mathcal{G}}$ is doubly stochastic, ${ }^{24}$

- $p_{i j}=0 \Longleftrightarrow q_{i j}=0$.

The coefficients $p_{i j}$ define a new linear updating rule applicable to the network $\mathcal{G} .^{25}$ Let us calculate the dynamics of the average of beliefs from one period to another according to this new updating rule:

$$
\begin{aligned}
\frac{1}{n} \sum_{i=1}^{n} y_{i}^{t+1} & =\frac{1}{n} \sum_{i=1}^{n} \sum_{j=1}^{n} p_{i j} y_{j}^{t} \\
& =\frac{1}{n} \sum_{j=1}^{n} \underbrace{\sum_{i=1}^{n} p_{i j}}_{=1} y_{j}^{t} \\
& =\frac{1}{n} \sum_{j=1}^{n} y_{j}^{t} .
\end{aligned}
$$

Such average is unchanged. By iterating this reasoning, we have that the average of opinions at any time is equal to the initial mean $\bar{x}$. When a consensus is reached, it is by definition the correct consensus.

Proof of Theorem 2. Given a network $\mathcal{G}$, let $\overline{\mathcal{G}}$ be the corresponding undirected network (with adjacency matrix $\bar{q}_{i j}=1 \Longleftrightarrow q_{i j}+q_{j i}>0$ ), and $\mathcal{G}_{i}{ }^{\delta}$ the subnetwork of $\mathcal{G}$ restricted to nodes which are distant at most $\delta \in \mathbb{N}$ from $i$ in $\overline{\mathcal{G}}$. Since the weights $\bar{\pi}_{i j}$ only depend on local properties of the network, there must exist a $\bar{\delta} \in \mathbb{N}$ such that they are only determined based on $\mathcal{G}_{i}^{\bar{\delta}}$. Consider then the networks in figure 7 :

\footnotetext{
${ }^{22}$ An $n \times n$ matrix $q$ is reducible if the set $\{1, \ldots, n\}$ can be partitioned in two subsets $V_{1}, V_{2}$ such that $q_{i j}=0$ whenever $i \in V_{1}$ and $j \in V_{2}$. This implies that nodes in $V_{1}$ are not connected to nodes in $V_{2}$, and hence that the network is not strongly connected.

${ }^{23}$ It is easy to see that if a network structure is strongly connected, statement $(i i)$ in their Theorem 1 holds. The matrix representation which they denote by $(*)$ implies that there are $k$ nodes with no links to some other $n-k$ nodes. But since in our network each agent is by assumption linked to herself, those two sets of nodes would have to be disjoint, and partition the whole network. Hence, there would be no path from the first to the second.

${ }^{24} \mathrm{~A}$ matrix is doubly stochastic if all its elements are non negative, and each row and column sums up to 1 .

${ }^{25}$ The relation between the double stochasticity of the updating matrix and the correctness of the consensus was already recognized by Harary (1959). The last part of this proof generalizes his Theorem 14 to a generic linear rule.
} 
Figure 7: Locally similar networks for node $E$

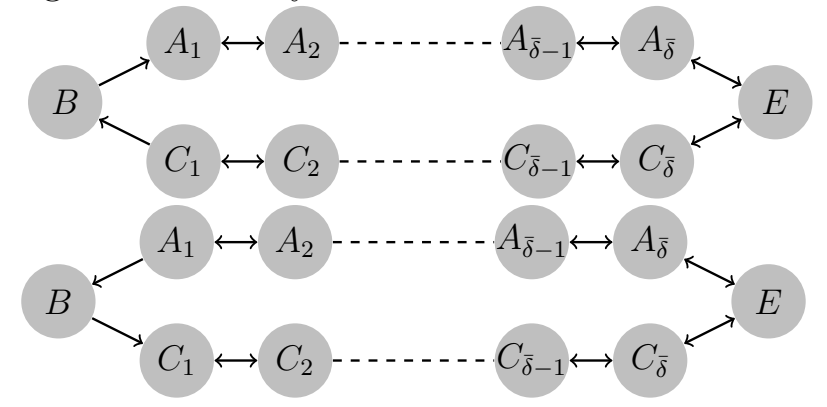

Notice that $\mathcal{G}_{E}^{\bar{\delta}}$ is identical in both networks, and hence the vector of weights $\bar{\pi}_{E}$ adopted by $E$ must be identical too. Assume, without loss of generality, that $\bar{\pi}_{E A_{\bar{\delta}}} \geq \bar{\pi}_{E C_{\bar{\delta}}}$. In the second network, $E$ gets to know the opinion of agents $B, C_{1}, \ldots, C_{\delta}$ only through the link coming from $C_{\delta}$. Hence, she is weighting the opinions of those $\bar{\delta}+1$ nodes less than the opinions of the other $\bar{\delta}$ nodes $A_{1}, \ldots, A_{\bar{\delta}}$. This rule cannot lead to the correct consensus on such network.

Example 1 Consider a network $\mathcal{G}$, with agents numbered from 1 to $n$, and execute the following steps:

$i$ start from agent 1: since the network is strongly connected, there must be a path from 1 to 2: let us denote it as $s_{1}$, and assume without loss of generality that it has no cycles;

ii again, since the network is strongly connected, there must be a path from 2 to 3: let us assume without loss of generality that it has no cycles, and denote as $s_{2}$ the union of $s_{1}$ with such a path;

iii by repeating the step above, for each $i<n$, a path $s_{i}$ is constructed, which goes from 1 to $i$ and passes through any $i^{\prime}<i$ : let $s_{n}$ be the union of $s_{n-1}$ with a path (again, without cycles) from $n$ to $1: s_{n}$ is a cycle which passes through each node at least once and at most $n$ times;

$i v$ for each pair $(i, j)$ with $j \neq i$, let $\pi_{i j}$ be defined as $\frac{1}{n}$ multiplied by the number of times that $s_{n}$ passes through the link from $j$ to $i$ (possibly zero). For each $i$, define $\pi_{i i}$ as $1-\sum_{j \neq i} \pi_{j i}$.

The updating rule having such $\pi_{i j}$ as updating weights is a valid linear rule. If we consider $s_{n}$ as a weighted network where the weight of a link is given by $\pi_{i j}$, it is strongly connected, and its adjacency matrix is doubly stochastic. Hence, the resulting updating rule leads to the correct consensus. 
Proof of Theorem 3. Consider the influence weights for the opinion of $a$ given agent in a given instant in time as a vector in the standard $n$-simplex, $\left\{\omega_{i j}^{t}\right\}_{j \leq n}$. For instance,

$$
\omega_{i}^{0}=(\underbrace{0, \ldots, 0}_{i-1}, 1, \underbrace{0, \ldots, 0}_{n-i})
$$

that is, before receiving any information from neighbors, the opinion of each agent is entirely formed by her initial signal. With a linear belief updating rule, the evolution of such vectors is simply described as a straightforward rewriting of equation (1):

$$
\begin{aligned}
\omega_{i}^{t} & =\sum_{j=1}^{n} q_{i j} \pi_{i j} \omega_{j}^{t-1} \\
& =\pi_{i i} \omega_{i}^{t-1}+\sum_{j \neq i} q_{i j} \pi_{i j} \omega_{j}^{t-1} .
\end{aligned}
$$

Since the network is anonymous, all neighbors of a given node are equivalent; and since the rule is anonymous, each node places an equal weight on each neighbor. So the above can be rewritten as

$$
\omega_{i}^{t}=\pi_{i i} \omega_{i}^{t-1}+\frac{\left(1-\pi_{i i}\right)}{d_{i}} \sum_{j \neq i} q_{i j} \omega_{j}^{t-1} .
$$

Notice that $y_{i}^{t}=\omega_{i}^{t} \cdot x$, and hence the beliefs are uniquely determined once $\pi_{i i}^{t}$ is fixed for each $i$.

For what concerns point 2 , let us define the system as biased at a given time $\bar{t}$ if for some $h$

$$
\sum_{i=1}^{n} \omega_{i h}^{\bar{t}} \neq 1
$$

Let us assume without loss of generality that $\bar{t}$ is the first time for which this happens. Notice that $\bar{t}>0$, since

$$
\sum_{i=1}^{n} \omega_{i h}^{0}=\omega_{h h}^{0}=1,
$$

and that

$$
\begin{aligned}
\sum_{i=1}^{n} \omega_{i h}^{\bar{t}} & =\sum_{i=1}^{n} \sum_{j=1}^{n} q_{i j} \pi_{i j} \omega_{j h}^{\bar{t}-1} \\
& =\sum_{j=1}^{n} \omega_{j h}^{\bar{t}-1} \sum_{i=1}^{n} q_{i j} \pi_{i j} .
\end{aligned}
$$


Since the network and the rule are anonymous, the value of the nested sum must be the same for all $j$, and since the sum of $q_{i j} \pi_{i j}$ across $j$ and $i$ is $n$, the value of such nested sum must be $\frac{n}{n}=1$. So

$$
\sum_{j=1}^{n} \omega_{j h}^{\bar{t}-1}=\sum_{i=1}^{n} \omega_{i h}^{\bar{t}} \neq 1 .
$$

But this contradicts the hypothesis that $\bar{t}$ is the first time at which the system is biased.

Proof of Theorem 4. One implication is obvious: given any agent $i$, let $\bar{d}$ be the (equal, by assumption) indegree of all $d_{i}+1$ agents in $S(i)$. Then, by applying equation (3), we have that for each $j \in S(i)$,

$$
\pi_{i j}=\frac{\bar{d}^{\rho}}{\left(d_{i}+1\right) \bar{d}^{\rho}}=\frac{1}{d_{i}+1},
$$

that is, the updating weights do not depend on the value of $\rho$ - hence neither the consensus belief nor social influence weights do. For the reverse implication, notice that there is at least a pair of agents $i, j$ with different indegree and such that $i$ listens to $j$ (if this is not the case, it is easy to show by induction that the network is regular). Define now as $H_{k}$ the set of agents $h$ such that there exist $j^{\prime}, j^{\prime \prime} \in S(h)$ with $k=d_{j^{\prime}}<d_{j^{\prime \prime}}$. Intuitively, we are considering all agents $i$ whose updating weights are affected by $\rho$ (because the neighbors have different indegree), and classifying them based on the lowest indegree of a neighbor: the underlying idea of the remaining of the proof is that this will allow us to identify an agent who is necessarily disadvantaged, in terms of social influence, by a strictly positive value of $\rho$. Let $\bar{k}$ be the smallest $k$ such that $H_{k}$ is non-empty - the non-regularity assumption means precisely that there exists at least one such $k$. Let $\bar{i} \in H_{\bar{k}}$, and $\bar{j} \in S(i)$ such that $d_{\bar{j}}=\bar{k}$. Notice that, for each $i^{\prime} \in S^{-1}(\bar{j}), \bar{j}$ must have smaller or equal indegree than all other agents in $S\left(i^{\prime}\right)$ (otherwise, we would have found a non-empty $H_{k^{\prime}}$ with $k^{\prime}<\bar{k}$ ). As a consequence, $\pi_{i^{\prime} \bar{j}}$ will be weakly smaller with $\rho>0$ than with $\rho=0$, and $\pi_{\bar{i} \bar{j}}$ will be strictly smaller. But since we know that

$$
w_{\bar{j}}=\sum_{i=1}^{n} \pi_{i \bar{j}} w_{i},
$$

this means that $w_{\bar{j}}$ will strictly decrease as a function of its neighbors, and hence that at least some $w_{i}$ will be affected by a change of $\rho$. 
Lemma A2 In a balanced network, when the GBR rule is applied with $\rho=0$, the social influence weight of each agent is proportional to her degree (including the self-link) $d_{i}+1 .^{26}$

Proof of Lemma A2. Recall from equation (8) that the vector of social influence weights is the unique element $w$ in $\mathbb{R}^{n}$ satisfying $\sum_{i=1}^{n} w_{i}=1$ and

$$
w_{i}=\sum_{j \in S^{-1}(i)} \pi_{j i} w_{j}
$$

when $\rho=0$, the above translates to

$$
w_{i}=\sum_{j \in S^{-1}(i)} \frac{1}{d_{j}+1} w_{j}
$$

Assuming the network is balanced, if the social influence weights can be written as $w_{i}=\alpha\left(d_{i}+1\right)$, where $\alpha$ is a constant, then the right hand side of equation (A.5) becomes

$$
\sum_{j \in S^{-1}(i)} \frac{1}{d_{j}+1} \cdot \alpha\left(d_{j}+1\right)=\left|S^{-1}(i)\right| \cdot \alpha=\alpha\left(d_{i}+1\right)=w_{i}
$$

that is, equation (A.4) is satisfied for each $i$. To guarantee that the sum of social influence weights adds up to 1 , it is sufficient to set $\alpha$ accordingly:

$$
\sum_{i=1}^{n} w_{i}=1 \Longrightarrow \alpha=\frac{1}{n+\sum_{i=1}^{n} d_{i}}
$$

(notice that such $\alpha$ is the inverse of the total number of links, including self-links).

Lemma A3 Given a network $\mathcal{G}$, let $\mathcal{E}_{\mathcal{G}}: \mathbb{R} \rightarrow(-1,0]$ be the function mapping each real number to the efficiency of the GBR rule played with such value of $\rho$. If $\mathcal{G}$ is balanced, $\frac{\partial \mathcal{E}_{\mathcal{G}}}{\partial \rho}(0)<0$.

Proof of Lemma A3. Lemma A2 tells us that on a balanced network, when $\rho=0$, influence weights are an increasing function of degree. Now consider a generalization of the GBR model in which each node $i$ adopts a different $\rho_{i}$, and let $\mathcal{E}_{\mathcal{G}}^{*}: \mathbb{R}^{n} \rightarrow(-1,0]$ be the corresponding generalization of $\mathcal{E}_{\mathcal{G}}$. We want to study $\mathcal{E}_{\mathcal{G}}^{*}$ around $(0, \ldots, 0)$. For small positive variations of a single $\rho_{\bar{i}}$, the elements of $S^{-1}(\bar{i})$ will be affected by a change in their

\footnotetext{
${ }^{26}$ This result was already proved by DeMarzo et al. (2003) (as part of their Theorem 6) for the specific case of undirected networks.
} 
social influence weight which is an increasing function of degree (and hence of social influence weights themselves), so that the sum of (squares of) absolute differences from the mean, $\frac{1}{n}$, will increase. Now, it may be that the indirect changes (i.e. on agents connected to agents in $\left.S^{-1}(i)\right)$ affect social influence weights in a way that is not an increasing function of degree. However, it is easy to see that the entity of such indirect changes will be smaller, in absolute value, than the direct change they originate from. So the same will hold for the squares of such differences, and as a result

$$
\frac{\partial \mathcal{E}_{\mathcal{G}}^{*}}{\partial \rho_{\bar{i}}}(0, \ldots, 0)<0
$$

Now, the derivative of $\mathcal{E}_{\mathcal{G}}$ in $\rho$ coincides with the directional derivative of $\mathcal{E}_{\mathcal{G}}^{*}$ along the vector $u=\left(\frac{1}{\sqrt{N}}, \ldots, \frac{1}{\sqrt{N}}\right)$ :

$$
\frac{\partial \mathcal{E}_{\mathcal{G}}}{\partial \rho}(0)=\nabla_{u} \mathcal{E}_{\mathcal{G}}^{*}(0, \ldots, 0)=\frac{1}{\sqrt{N}} \sum_{i=1}^{N} \frac{\partial \mathcal{E}_{\mathcal{G}}^{*}}{\partial \rho_{i}}(0, \ldots, 0)<0
$$

Proof of Theorem 5. It is easy to verify that $\mathcal{E}_{\mathcal{G}}$ is smooth. Assume then that there exists some $\rho^{*}>0$ such that $\mathcal{E}_{\mathcal{G}}\left(\rho^{*}\right)>\mathcal{E}_{\mathcal{G}}(0)$ : since (by Lemma A3) $\mathcal{E}_{\mathcal{G}}^{\prime}(0)<0$, it must be that $\mathcal{E}_{\mathcal{G}}$ has a local minimum $\rho_{*} \in\left[0, \rho^{*}\right]$. However, since $\rho_{*}>0$, the derivative in $\rho$ of a given term $\left(w_{i}-\frac{1}{n}\right)^{2}$ is larger the larger $w_{i}$. So if the linear combination of such derivatives is 0 in $\rho_{*}$, it must be positive in a right neighborhood of $\rho_{*}$, that is, $\mathcal{E}_{\mathcal{G}}^{\prime \prime}\left(\rho_{*}\right)<0$. So $\rho_{*}$ is not a local minimum.

Proof of Theorem 6. It is easy to verify that in $\mathcal{G}^{\prime}$, the node $h$ (respectively: $i$ ) has, under $\rho=0$, a lower (respectively: higher) influence weight than any node in $S^{-1}(h) \backslash S(h)$ (respectively: $S^{-1}(i) \backslash S(i)$ ) (which is necessarily non-empty). Let us now analyze the step-by-step effect of a marginal increase in $\rho$. After the first step of the updating process, it simply results in an increase (respectively: decrease) of social influence for $h$ (respectively: $i$ ), at the expense of nodes in the listening sets of nodes in $S^{-1}(h)$. After the second step, it will additionally increase (respectively: decrease) the social influence weights of all nodes in $S(h)$ (respectively: $S(i)$ ). Both these effects strictly increase efficiency. In any subsequent step $K$, the higher (respectively: lower) weights applied to information coming from $h$ (respectively: $i$ ) will have as additional effects a relative increase (respectively: decrease) of influence for nodes $l$ such that there is a path of length less than $K$ from $l$ to $h$ (respectively: $i$ ). However, in the composition of the beliefs of $h$ (respectively: of $i$ ), the opinion of such nodes has a lower weight than the opinion of 
any node in $S(h)$ (respectively: in $S(i)$ ). As a consequence, there is a $\bar{\rho}>0$ such that for any $\rho \in(0, \bar{\rho})$, the increases of influence for nodes in $S(h)$ and the decreases of influence for nodes in $S(i)$ are larger than any other variations in social influence weights. In such interval, the GBR is guaranteed to perform strictly better than with $\rho=0$.

Proof of Theorem 7. Given a natural number $K$, consider a network having the following binary tree-like structure:

- an agent $A_{1}$ listens to two other agents $A_{2,1}, A_{2,2}$,

- each agent $A_{k, i}$ listens to two other agents $A_{k+1,2 i-1}, A_{k+1,2 i}$, for each $k<K$,

- each "leaf" agent $A_{K, i}$ listens to $A_{1}$ and to her "close relatives" $A_{K, i-1}$ and $A_{K, i+1}\left(A_{K, 1}\right.$ listens to $A_{K, 2^{K}}$ and $A_{K, 2}$, while $A_{K, 2^{K}}$, listens to $A_{K, 2^{K}-1}$ and $\left.A_{K, 1}\right)$.

Notice that,

- the structure is perfectly symmetric, in the sense that all the agents positioned on a given "layer" will exhibit the same vector of updating weights (which for simplicity we will denote respectively as $\pi_{k-1, k}$ and $\pi_{k, k}$, rather than $\pi_{A_{k-1, i} A_{k, j}}$ and $\left.\pi_{A_{k, i} A_{k, i}}\right)$ and the same social influence (which we will hence denote as $w_{k}$ rather than $w_{A_{k, i}}$ ),

- for most of the layers of this structure, the updating weights are independent from $\rho$; namely, for any $k$ such that $1 \leq k<K-1$,

$$
\pi_{k, k}=\pi_{k-1, k}=\frac{2^{\rho}}{3 \cdot 2^{\rho}}=\frac{1}{3}
$$

and hence

$$
\begin{aligned}
w_{k} & =\pi_{k, k} w_{k}+\pi_{k-1, k} w_{k-1} \\
& =\frac{1}{3} w_{k}+\frac{1}{3} w_{k-1} \\
& =\frac{1}{2} w_{k-1}
\end{aligned}
$$

- since the number of agents on a given layer doubles at each level, the sum of social influences of all agents in a given layer, which we will denote as $W_{k}$, is the same for any $k$ from 1 to $K-1$. 
Now consider the social weight of $A_{1}$. The updating weights adopted by a leaf (which has indegree 3, rather than 2) can be calculated as:

$$
\pi_{K, 1}=\frac{2^{\rho}}{2^{\rho}+3^{\rho}+3^{\rho}+3^{\rho}}=\frac{2^{\rho}}{2^{\rho}+3^{\rho+1}} ; \quad \pi_{K, K}=\frac{3^{\rho}}{2^{\rho}+3^{\rho+1}} .
$$

and since $2^{K}$ leafs listen to $A_{1}$,

$$
\begin{aligned}
w_{1} & =\pi_{1,1} w_{1}+2^{K} \pi_{K, 1} w_{K} \\
& =\frac{1}{3} w_{1}+2^{K} \frac{2^{\rho}}{2^{\rho}+3^{\rho+1}} w_{K} \\
& =2^{K} \frac{3 \cdot 2^{\rho-1}}{2^{\rho}+3^{\rho+1}} w_{K} \\
\Longrightarrow W_{1} & =\frac{3 \cdot 2^{\rho-1}}{2^{\rho}+3^{\rho+1}} W_{K} .
\end{aligned}
$$

Assume the optimal level of $\rho$ is bounded above by some $\hat{\rho}$. This means that for $K \rightarrow \infty$, this last ratio will also be bounded by positive numbers from above and from below. That is, asymptotically,

$$
W_{K} \sim W_{1}=W_{2}=\cdots=W_{K-1}
$$

and hence, since the sum of all $w_{i}$ is $1, w_{1}=W_{1}$ will converge to 0 asymptotically as $\frac{\alpha}{K}$, where $\alpha$ is a constant. Now, it easy instead to verify that since, for given $\bar{K}$,

$$
2^{\bar{K}} \frac{3 \cdot 2^{\rho-1}}{2^{\rho}+3^{\rho+1}} \stackrel{\rho \rightarrow \infty}{\rightarrow} 0
$$

and such term is continuous in $\rho$, we can define $\rho_{\bar{K}}$ such that

$$
2^{\bar{K}} \frac{3 \cdot 2^{\rho_{\bar{K}}-1}}{2^{\rho_{\bar{K}}}+3^{\rho_{\bar{K}}+1}}=1
$$

moreover, it is straightforward to verify that $\rho_{\bar{K}} \stackrel{\bar{K} \rightarrow \infty}{\rightarrow} \infty$. When the GBR rule is applied with such $\rho_{K}$, we have, by definition, that $w_{1}=w_{K}$. That is,

$$
\frac{W_{K}}{2^{K}}=W_{1}=W_{1}=W_{2}=\cdots=W_{K-1} .
$$

The nodes with maximum influence are now $2^{K}+1$ (all leafs, and $A_{1}$ ) so each influence weight will converge to 0 as $\frac{1}{2^{K}}$ (or faster), rather than as $\frac{\alpha}{K}$.

Now, observe that the correct weights converge to 0 as $\frac{1}{n}=\frac{1}{2^{K+1}-1}$. Hence, the sum of square deviations in the case of any finite $\rho$ will converge towards at least $\left(\frac{\alpha}{K}\right)^{2}=\frac{\alpha^{2}}{K^{2}}$, while in the case of $\rho=\rho_{K}$ it will converge towards at most

$$
2^{K+1} \cdot\left(\frac{1}{2^{K}}\right)^{2}=\frac{2^{K+1}}{2^{2 K}}=\frac{1}{2^{K-1}} \stackrel{K \rightarrow \infty}{<} \frac{\alpha^{2}}{K^{2}} .
$$

Hence the most efficient $\rho$ for $K \rightarrow \infty$ must also tend to $\infty$. 


\section{Appendix B - Experimental Instructions}

[Translated from Italian]

Welcome and thank you for taking part in this experiment. During the experiment talking or communicating with other participants is not allowed in any way. If you have a question at any time, raise your hand and one of the assistants will come to answer your question. By carefully following the instructions you can earn a sum of money that will depend on the choices made by you and the other participants. On top of that amount, you will receive in any case $5 €$ for the participation in this experiment.

\section{General Rules}

- 24 subjects will take part in this experiment.

- The experiment takes place in 4 phases of 8 rounds each, for a total of 32 rounds.

- At the beginning of the experiment 6 groups of four subjects will be randomly and anonymously formed by the computer.

- You will be assigned to one of the 6 groups. You will interact only with those in your group, without knowing their identity. The composition of each group will remain unchanged throughout the experiment.

\section{The development of a phase}

- In the first round of each of the four phases, in all groups, each subject will be randomly and anonymously assigned a different role: A, B, C, and D.

- The computer will randomly generate four integers that we will refer to as signals. Each component of the group will be shown only one of the four signals. Signals will be denoted as $x_{A}, x_{B}, x_{C}$, and $x_{D}$.

- In each of the 8 periods of the phase, each subject will be asked to guess the mean of the four signals extracted by the computer for that phase: $\bar{x}=\frac{\left(x_{A}+x_{B}+x_{C}+x_{D}\right)}{4}$.

- For making each guess, there is a maximum time of 120 seconds (which will be shown by a counter in the top right corner of the screen).

- At any moment, it is possible to open a calculator by simply clicking its icon, in the bottom left corner of the screen.

\section{How earnings are determined}


- Individual earnings will depend on how close the guess comes to the value of $\bar{x}$ :

- At the end of the experiment, the computer will randomly extract one of the 32 periods.

- The earnings will be equal to 15 euros minus the difference (in absolute value) between $\bar{x}$ and the guess made in the selected round.

- If this difference turns out to be negative, the subject will earn 0 euros.

- Examples:

- if $\bar{x}=1424$ and the guess is 1424 , the difference is 0 and earnings are 15 euros.

- if $\bar{x}=308$ and the guess is 311.5 , the difference is 3.5 and earnings are 11.5 euros.

- if $\bar{x}=803.25$ and the guess is 792 , the difference is 11.25 and earnings are 3.75 euros.

- if $\bar{x}=62.5$ and the guess is 30.5 , the difference is 32 and earnings are 0 euros, since $15-32<0$.

In each of this cases, the participant will also receive 5 euros for participating in the experiment.

- In each round, the optimal guess (which allows to get the maximum earnings) depends on the information that each subject has on the signals:

- if she knows only her own signal, the optimal choice is her own signal,

- if she knows or can infer two signals, her optimal choice is the mean of the two signals;

- if she knows or can infer three signals, her optimal choice is the mean of the three signals;

- if she knows or can infer four signals, her optimal choice is the mean of the four signals.

\section{Information}

- In each of the tree phases 
- In the first round, each subject knows her own signal.

- From the second round onwards, before making his choice, each subject will be informed by the computer of the choices made in the previous rounds by some of the components of her group, based on the structure represented in the following figure:

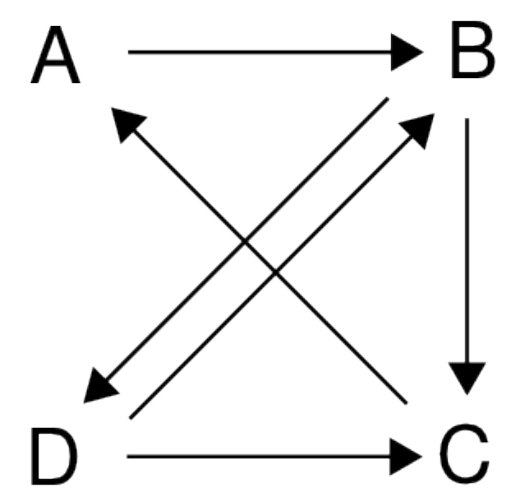

- Therefore, before making his choice

- A will be informed of the choices made by $\mathrm{C}$.

- B will be informed of the choices made by A and D.

- $\mathrm{C}$ will be informed of the choices made by B and D.

- D will be informed of the choices made by $\mathrm{B}$.

- The roles (A, B, C, D), the signals $\left(x_{A}, x_{B}, x_{C}, x_{D}\right)$ and by consequence their mean will change at each phase: the computer will generate them randomly before the first period of the phase.

\section{Feedback and payments}

- At the end of each phase the computer will show to each subject the four signals of his group, their mean, and the choices made.

- At the end of the experiment each subject will be shown the round the computer has selected to determine payments, the value of $\bar{x}$ for her group, the choice she made and the corresponding amount earned in euro.

- The experiment will terminate and the amount earned by each subject will be paid in cash.

\section{Control questions}


1. If you knew only your signal $(a)$, what would be your optimal guess?

2. If you knew your signal $(a)$ as well as the one of another member of your, group $(b)$, what would be your optimal guess?

3. If you knew your signal $(a)$ as well as the ones of two other members of your group $(b$ and $c$ ), what would be your optimal guess? ...............

4. If you knew your signal $(a)$ as well as the ones of three other members of your group $(b, c$, and $d)$, what would be your optimal guess? 


\section{References}

Acemoglu, D., Bimpikis, K., Ozdaglar, A., 2014. Dynamics of information exchange in endogenous social networks. Theoretical Economics 9 (1), 4197.

Acemoglu, D., Dahleh, M. A., Lobel, I., Ozdaglar, A., 2011. Bayesian learning in social networks. The Review of Economic Studies 78 (4), 1201-1236.

Acemoglu, D., Ozdaglar, A., 2011. Opinion dynamics and learning in social networks. Dynamic Games and Applications 1 (1), 3-49.

Bala, V., Goyal, S., 1998. Learning from neighbours. The Review of Economic Studies 65 (3), 595-621.

Baños, R. A., Borge-Holthoefer, J., Moreno, Y., 2013. The role of hidden influentials in the diffusion of online information cascades. EPJ Data Science $2(1), 1-16$.

Bonacich, P., 1972. Factoring and weighting approaches to status scores and clique identification. Journal of Mathematical Sociology 2 (1), 113-120.

Buechel, B., Hellmann, T., Klößner, S., 2014. Opinion dynamics and wisdom under conformity. Journal of Economic Dynamics and Control, Forthcoming.

Corazzini, L., Pavesi, F., Petrovich, B., Stanca, L., 2012. Influential listeners: An experiment on persuasion bias in social networks. European Economic Review 56 (6), 1276-1288.

DeGroot, M. H., 1974. Reaching a consensus. Journal of the American Statistical Association 69 (345), 118-121.

DellaVigna, S., Gentzkow, M., 2010. Persuasion: Empirical evidence. Annual Review of Economics 2, 643-669.

DeMarzo, P. M., Vayanos, D., Zwiebel, J., 2003. Persuasion bias, social influence, and unidimensional opinions. The Quarterly Journal of Economics 118 (3), 909-968.

Fischbacher, U., 2007. z-tree: Zurich toolbox for ready-made economic experiments. Experimental economics 10 (2), 171-178.

French, Jr, J. R., 1956. A formal theory of social power. Psychological review $63(3), 181$. 
Friedkin, N. E., Johnsen, E. C., 1990. Social influence and opinions. Journal of Mathematical Sociology 15 (3-4), 193-206.

Gale, D., Kariv, S., 2003. Bayesian learning in social networks. Games and Economic Behavior 45 (2), 329-346.

Gleeson, J. P., Cellai, D., Onnela, J.-P., Porter, M. A., Reed-Tsochas, F., 2014. A simple generative model of collective online behavior. Proceedings of the National Academy of Sciences 111 (29), 10411-10415.

URL http://www.pnas.org/content/111/29/10411. abstract

Golub, B., Jackson, M. O., 2010. Naïve learning in social networks and the wisdom of crowds. American Economic Journal: Microeconomics 2 (1), $112-149$.

Harary, F., 1959. A criterion for unanimity in French's theory of social power. In: Cartwright, D. (Ed.), Studies in social power. University of Michigan.

Hegselmann, R., Krause, U., 2002. Opinion dynamics and bounded confidence models, analysis, and simulation. Journal of Artificial Societies and Social Simulation 5 (3).

Jackson, M. O., 2010. Social and economic networks. Princeton University Press.

Jackson, M. O., Yariv, L., 2010. Diffusion, strategic interaction, and social structure. In: Benhabib, J., Bisin, A., Jackson, M. O. (Eds.), Handbook of Social Economics. Elsevier.

Jadbabaie, A., Molavi, P., Sandroni, A., Tahbaz-Salehi, A., 2012. Nonbayesian social learning. Games and Economic Behavior 76 (1), 210-225.

Möbius, M., Phan, T., Szeidl, A., 2010. Treasure hunt. Working Paper 1343, Society for Economic Dynamics.

Olfati-Saber, R., Murray, R. M., 2004. Consensus problems in networks of agents with switching topology and time-delays. Automatic Control, IEEE Transactions on 49 (9), 1520-1533.

Perfect, H., Mirsky, L., 1965. The distribution of positive elements in doublystochastic matrices. Journal of the London Mathematical Society 1 (1), 689-698.

Sridharan, M., Parthasarathy, K., 1972. Isographs and oriented isographs. Journal of Combinatorial Theory, Series B 13 (2), 99-111. 